\title{
Analysis of the ITER low field side reflectometer employing the Beam Tracing method
}

\author{
A. Stegmeir ${ }^{a, *}$, G.D. Conway ${ }^{\mathrm{a}}$, E. Poli ${ }^{\mathrm{a}}$, E. Strumberger ${ }^{\mathrm{a}}$ \\ ${ }^{a}$ Max-Planck-Institut für Plasmaphysik, Boltzmanstrasse 2, D-85748 Garching bei München, Germany
}

\begin{abstract}
The Beam Tracing Method, which describes electromagnetic wave propagation in the short wavelength limit including diffraction effects, is applied to microwave reflectometry. The TORBEAM code has been augmented with relativistic corrections to the electron mass - which are necessary for a reliable description in high temperature plasmas such as in ITER, and a beam coupling model for the receiving antenna coupling. The propagation and reception behaviour of reflectometer probe beams in the ITER geometry is computed. The received power is affected by the intensity of the beams, the offset of the beams respectively to the receiver antenna and the angle of incidence. Using a magnetic field derived from a 3D equilibrium it is shown that the effects of toroidal field ripple in ITER on the beam propagation are negligible. Various antenna configurations for the ITER low field side reflectometer are proposed and analyzed, particularly their sensitivity to plasma height variations.
\end{abstract}

Keywords: Beam Tracing, Reflectometry, ITER, field ripple

\footnotetext{
*Corresponding author

Email address: stegmeia@ipp.mpg.de (A. Stegmeir)

${ }^{1}$ Tel.: +49 89 32991598; fax: +49 8932992580
} 


\section{Introduction}

Microwave reflectometry is a widely used diagnostic technique for studying magnetically confined plasmas. A microwave/millimeter-wave beam is launched into the plasma (usually perpendicular to the confining magnetic field and parallel to the density gradient) where it propagates until it reaches the cutoff condition (where the plasma refractive index goes to zero) and is reflected. The refractive index depends on the probing microwave frequency $\omega$, the plasma density $n_{e}$, and (for X-mode polarization) the magnetic field $B$, and hence different parts of the plasma can be probed by varying the microwave frequency. By measuring the phase delay of reflected beam information on the radial profile of the electron density and its fluctuations can then be obtained [1, 2].

On ITER several reflectometer diagnostic systems are being developed to measure the edge and core density profiles, the density fluctuations as well as the plasma position and rotation $[3,4]$. The primary system is the so-called Low-field-Side Reflectometer (LFSR) system which consists of several reflectometers probing the plasma mid-plane from the tokamak outer, or low magnetic field side. The LFSR system design has evolved steadily in recent years $[4,5,6,7]$. The most challenging aspect of the system is the design of the 'front-end' components - the antennas and waveguide transmission lines which will be installed in the vacuum vessel port-plug. The number of antennas, their position, orientation and size (i.e. gain) will critically affect the diagnostic's ability to meet the measurement requirements [4]. Hence careful design of these components, based principally on simulation studies, will be crucial to the success of the diagnostic.

In this paper a detailed investigation of the basic behaviour of the beam propagation dependence on the antenna parameters is presented using the TORBEAM beam tracing code (which includes diffraction effects and relativistic corrections) with simulated ITER magnetic equilibrium and density and temperature profiles. Several possible antenna configurations are investigated using the beam tracing code coupled with antenna power coupling calculations. This allows a much more rigorous assessment and comparison of their relative performance and merits than was previously available [7].

The paper begins with a description of the computational model used, including the modeling of the launched beam, a background to the beam-tracing equations, and the formulation of the beam coupling efficiency from which the received power is calculated. An outline of the LFSR operational range together with details of the ITER configuration then follow. The criteria for the assessment exercise are then presented. The results are divided into two sections: first the basic behaviour of a single launched microwave probe beam is described and how it depends on the plasma geometry and the launch antenna parameters. Next, a detailed assessment of specific transmit (launch) and receive antenna configurations, such as monostatic, bistatic, hybrids, antenna arrays and their variations, is presented.

\section{Computational model}

\subsection{Modeling of launched beam}

The ITER LFSR system will use circular aperture type antennas embedded into the equatorial port-plug blanket module. The antennas will be fed by low-loss oversized corrugated circular waveguide supporting the $\mathrm{HE}_{11}$ waveguide mode [8]. This mode couples in to an Gaussian beam antenna radiation pattern after a short distance $(\approx 1 / 3 \cdot$ Rayleigh length, compare fig.2 in [9]). Figure 1 shows the behaviour of the beam. In this frame, the normalized wave-function of the launch/receiver antenna radiation pattern $\chi$ follows from the vacuum solution for Gaussian beams:

$$
\chi(\vec{r})=\frac{\sqrt{2 / \pi}}{w_{\chi}(x)} \exp \left[-i K x-i \frac{K}{2} \frac{y^{2}+z^{2}}{R_{\chi}(x)}-\frac{y^{2}+z^{2}}{w_{\chi}^{2}(x)}\right],
$$

where the beam width, which is the radius of the $1 / e^{2}$ contour of the intensity cross section, and phase front curvature are [10]:

$w(x)=w_{0}\left[1+\left(\frac{x}{x_{R}}\right)^{2}\right]^{1 / 2}, \quad R(x)=x\left[1+\left(\frac{x_{R}}{x}\right)^{2}\right]$ 
with $x$ the distance from the antenna and $x_{R}=$ $w_{0}^{2} \omega / 2 c$ the Rayleigh length. The phase-front radius of curvature at the antenna aperture is usually given by the slant length of the antenna horn [10]. For the ITER antennas the slant length will be very long, so a good approximation is to set the phase-front curvature at the antenna aperture to infinity (i.e. flat phase-front). Hence the waist of the launched beam, which is given by $w_{0}=0.32 \mathrm{D}$, is directly at the antenna aperture. At large distances $x \gg x_{R}$ the following approximations hold:

$$
w(x) \approx x \tan \theta_{\text {div }}, \quad R(x) \approx x,
$$

with the divergence angle $\tan \theta_{\text {div }}=2 c / \omega w_{0}$. Thus, after a few Rayleigh lengths the size of the cross section is determined by the divergence angle. The lower the frequency and the smaller the diameter of the antenna the larger the cross section.

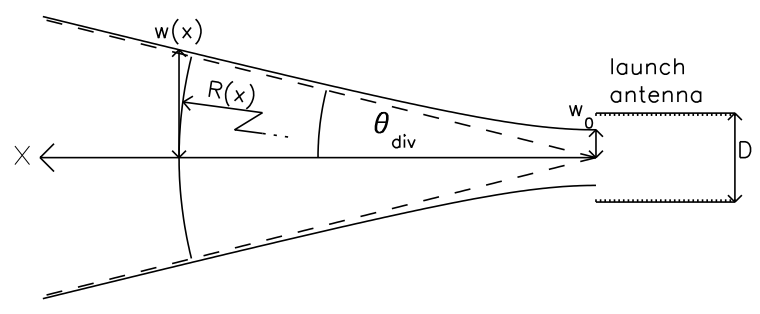

Figure 1: Launch of Gaussian beam from corrugated waveguide.

\subsection{Beam Tracing}

The propagation of the Gaussian beam through the plasma is calculated using the beam tracing approach $[11,12]$. Beam tracing is superior to Ray-tracing $[13,14]$ since it retains diffraction effects, and yields an analytic expression for the electric field across the beam profile, which is also possible, but not straightforward with Ray-tracing [15]. Beam tracing is also computationally fast compared to full-wave simulations, since only ordinary differential equations need to be solved instead of partial ones.

Beam tracing gives an approximate solution of the Maxwell equations in weakly inhomogeneous media for wave-beams satisfying the condition:

$$
\lambda \ll w \ll L,
$$

where $\lambda$ is the probing wavelength and $L$ the typical inhomogeneity scale length of the medium. In the beam tracing solution the propagation of the wave is described by a central ray, which obeys the same laws as in geometric optics:

$$
\frac{d q_{\alpha}}{d \tau}=\frac{\partial H^{M}}{\partial k_{\alpha}}, \quad \frac{d K_{\alpha}}{d \tau}=-\frac{\partial H^{M}}{\partial x_{\alpha}},
$$

where $q_{\alpha}$ is the trajectory of the central ray, $K_{\alpha}$ the wavevector of the central ray and $H^{M}$ a solution of the dispersion function:

$$
\operatorname{det}\left[\frac{c^{2}}{\omega^{2}}\left(-k^{2} I+\vec{k} \vec{k}\right)+\epsilon^{h}\right]=0,
$$

with $\epsilon^{h}$ the hermitian part of the dielectric tensor. In this work the anti-hermitian part is assumed to be zero, i.e. there is zero absorption. $\alpha($ and $\beta, \gamma)$ are indexes over the $x, y, z$ coordinates.

The electric field is expressed in terms of a complex phase $\bar{s}(\vec{r})=s(\vec{r})+i \phi(\vec{r})$ :

$$
\vec{E}(\vec{r})=\vec{A}(\vec{r}) e^{i s(\vec{r})-\phi(r)} .
$$

where $s(\vec{r})$ describes the phase of the beam and $\phi(\vec{r})$ describes a Gaussian cross section. The scale ordering in condition (4) allows a paraxial expansion, i.e. $s(\vec{r})$ and $\phi(\vec{r})$ are expanded up to second order around the central ray:

$s(\vec{r})=s_{0}(\vec{r})+K_{\alpha}\left[x_{\alpha}-q_{\alpha}\right]+\frac{1}{2} s_{\alpha \beta}\left[x_{\alpha}-q_{\alpha}\right]\left[x_{\beta}-q_{\beta}\right]$,

$\phi(\vec{r})=\frac{1}{2} \phi_{\alpha \beta}\left[x_{\alpha}-q_{\alpha}\right]\left[x_{\beta}-q_{\beta}\right]$.

The matrix $s_{\alpha \beta}$ and the positive definite matrix $\phi_{\alpha \beta}$ have been introduced, where the complex quantity $\bar{s}_{\alpha \beta}=s_{\alpha \beta}+i \phi_{\alpha \beta}$ obeys a complex matrix Riccati differential equation:

$$
\begin{aligned}
\frac{d \bar{s}_{\alpha \beta}}{d \tau}= & -\left(\frac{\partial^{2} H^{M}}{\partial x_{\alpha} \partial x_{\beta}}+\frac{\partial^{2} H^{M}}{\partial x_{\beta} \partial k_{\gamma}} \bar{s}_{\alpha \gamma}\right. \\
& \left.+\frac{\partial^{2} H^{M}}{\partial x_{\alpha} \partial k_{\gamma}} \bar{s}_{\beta \gamma}+\frac{\partial^{2} H^{M}}{\partial k_{\gamma} \partial k_{\delta}} \bar{s}_{\alpha \gamma} \bar{s}_{\beta \delta}\right)\left.\right|_{\substack{x_{\alpha}=q_{\alpha} \\
k_{\alpha}=K_{\alpha}}} .
\end{aligned}
$$


The second order expansion parameter of eq. (8), the matrix $s_{\alpha \beta}$, is related with the curvature radius $R$ of the phase front and the second order expansion parameter of eq. (9), the matrix $\phi_{\alpha \beta}$, is related with the width $w$ of the beam, at which the intensity of the beam drops to $1 / e^{2}$ compared to the intensity at the central ray:

$$
s_{\alpha \beta} \sim \frac{\omega / c}{R}, \quad \phi_{\alpha \beta} \sim \frac{2}{w^{2}} .
$$

Analytic solutions of the beam tracing equations in a slab model are presented in $[16,17,18]$.

The TORBEAM code [20] solves the beam tracing equations numerically in a tokamak geometry with experimentally prescribed magnetic equilibria, density and temperature profiles. TORBEAM uses the cold plasma approximation of the dielectric tensor to calculate the beam trajectory and has implemented a generalized Snell's law [21] to take into account the transition at the vacuum plasma boundary. In ITER relativistic effects are expected to be important due to the high core electron temperatures. This was incorporated into the code using a simple, but well established, approximation of an effective electron mass in the cold dielectric tensor [22]:

$$
m_{\mathrm{eff}}=m_{0}\left(1+5 \frac{k_{B} T_{e}}{m_{0} c^{2}}\right)^{1 / 2},
$$

where $m_{0}$ is the rest mass of the electron, $k_{B}$ the Boltzmann constant, $T_{e}$ the electron temperature and $c$ the speed of light. This approximation is in line with other models (e.g. [23]) and is sufficient for our investigations which are mainly constrained to the cooler $\left(T_{e} \leq 5 \mathrm{eV}\right)$ pedestal region. Mazzucato [22] has also demonstrated that this approximation describes well the relativistic effects on beam propagation to the cutoff. For ITER core probing a more relativistic correction model may be required.

Short wavelength approximations, such as Raytracing and Beam-tracing, break down at the cutoff, because here the wavelength tends to infinity and condition (4) is not fulfilled. A reconstruction of the wave field based on Beam tracing becomes inaccurate if the launched and reflected waves interfere significantly in the caustic region, i.e., for a beam close to perpendicular to the cutoff surface. However, comparisons of Beam-tracing with analytic solutions [24, 25] and full-wave simulations [26] show that the "classical" turning point, as predicted by Beam-tracing, differs only slightly from the position of the caustic as reconstructed from the exact field pattern. The asymptotic solution for the wave field far from the cutoff is also in good agreement with analytic solutions.

\subsection{Receiver coupling}

\subsubsection{Beam coupling efficiency}

In this section the power coupling efficiency between the reflected probe beam and the receiver antenna is derived. Figure 2 schematically shows the beam-antenna geometry. Defining the receiver antenna frame $(x, y, z)$ with the centre of the antenna aperture as the origin and the $x$-axis in direction of the antenna line-of-sight (LoS), the back extended normalized wave-function of the receiver antenna radiation pattern $\chi$ is given by eq. 1 . Let $\Psi$ be the normalized wave-function of the reflected beam, which can be written in terms of beam-tracing parameters:

$$
\begin{aligned}
\Psi(\vec{r})= & \left(\frac{2}{\pi w_{\Psi, \text { maj }} w_{\Psi, \text { min }}}\right)^{1 / 2} \\
& \exp \left[i K_{\alpha} r_{\alpha}+\frac{i}{2} s_{\alpha \beta}\left(r_{\alpha}-q_{\alpha}\right)\left(r_{\beta}-q_{\beta}\right)\right. \\
& \left.-\frac{1}{2} \phi_{\alpha \beta}\left(r_{\alpha}-q_{\alpha}\right)\left(r_{\beta}-q_{\beta}\right)\right]
\end{aligned}
$$

with $w_{\Psi, m a j}, w_{\Psi, \min }$ the major and minor widths of the reflected beam that can be deduced from the eigenvalues of the matrix $\phi_{\alpha \beta}$ according to relation 11.

The coupling efficiency is given by $[27,28]$ :

$$
T_{a}=\left|\int_{-\infty}^{\infty} \int_{-\infty}^{\infty} \Psi^{*}\left(x_{0}, y, z\right) \chi\left(x_{0}, y, z\right) d y d z\right|^{2}
$$

where the integration plane takes place at an arbitrary fixed $x_{0}$, which is chosen here as the receiver antenna plane $\left(x_{0}=0\right)$, i.e. $w_{\chi} \rightarrow w_{\chi, o}$ and $R_{\chi} \rightarrow \infty$. 


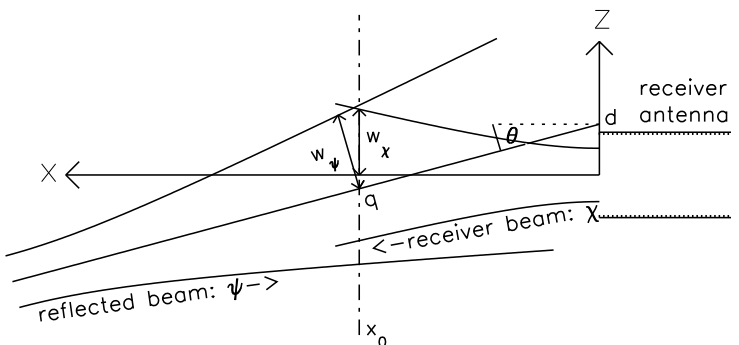

Figure 2: Schematic of coupling between reflected beam and receiver antenna.

Applying the rules for Gaussian integrals yields:

$$
\begin{aligned}
T_{a} & =\frac{16}{w_{\chi, 0}^{2} w_{\Psi, m a j} w_{\Psi, \min }} \frac{1}{\operatorname{det}(\Sigma+\sigma) \mid} \\
& \exp \left(-\phi_{\alpha \beta} q_{\alpha} q_{\beta}\right) \exp \left[\Re\left\{(\Sigma+\sigma)_{\alpha \beta}^{-1} b_{\alpha} b_{\beta}\right\}\right],
\end{aligned}
$$

where $\Sigma_{\alpha \beta}=\phi_{\alpha \beta}+i s_{\alpha \beta}$, and $\sigma_{\alpha \beta}=\frac{2}{w_{\chi, 0}^{2}} \mathbf{1}_{\alpha \beta}$ are two dimensional matrices and $b_{\alpha}=\Sigma_{\alpha \beta} q_{\beta}-i K_{\alpha}$ a vector, and the indexes $\alpha, \beta$ now only run over the $y, z$-components.

Equation 15 is the most generic formula for the beam coupling. It allows for a finite angle of incidence, an axial offset in incidence, as well as for an asymmetric beam shape. However, to illustrate the basic behaviour of the coupling it is easier turn to the simpler case of a symmetric beam (circular cross section and isotropic phase-front curvature) incident on the receiver antenna with an angle $\theta$ and an offset $d$. The coupling, which is derived in full in Appendix A together with some special cases, becomes, for small angles of incidence $(\theta \ll 1$ radian $)$ :

$$
\begin{aligned}
T_{a} & \approx T_{0} \exp \left\{-T_{0} / 2[\right. \\
& \left(d^{2} / w_{\chi, 0}^{2} w_{\Psi}^{2}+K^{2}\left(d / R_{\Psi}-\theta\right)^{2} / 4\right)\left(w_{\chi, 0}^{2}+w_{\Psi}^{2}\right) \\
& \left.\left.-K^{2} w_{\chi, 0}^{2} d\left(d / 2 R_{\Psi}-\theta\right) / 2 R_{\Psi}\right]\right\},
\end{aligned}
$$

where

$$
T_{0}=\frac{4}{\left(w_{\Psi} / w_{\chi, 0}+w_{\chi, 0} / w_{\Psi}\right)^{2}+K^{2} w_{\Psi}^{2} w_{\chi, 0}^{2} / 4 R_{\Psi}^{2}}
$$

is the coupling coefficient for a beam co-linear with the antenna. ¿From equation 16 it can be seen, that the coupling depends on the size of the reflected beam cross-section and the beam phase-front curvature (via $T_{0}$ ), and, exponentially on the angle of incidence $\theta$ and the offset $d$. Qualitatively, this means a high coupling can be achieved if the reflected footprint is not too large, but, has an adequate overlap with the receiver antenna, and if the reflected beam phasefront is parallel to the antenna plane.

\subsubsection{Gain Function}

The antenna - reflected beam coupling can also be approximated well by the classical antenna gain function. Here, the receiver antenna is treated as a point object and antenna received power $P_{r}$ is given as [29]:

$$
P_{r}=I \frac{\pi}{K^{2}} G\left(\theta_{i n c}\right),
$$

with $I$ the intensity of the beam at the centre of the receiver antenna. The antenna gain function is given by $[18]$ :

$$
G\left(\theta_{i n c}\right)=\frac{8}{\tan \theta_{d i v}} \frac{1}{\cos ^{3} \theta_{i n c}} \exp \left[-2 \frac{\tan ^{2} \theta_{i n c}}{\tan ^{2} \theta_{d i v}}\right] .
$$

Here, the angle of incidence $\theta_{i n c}$ is defined as the angle between the antenna line of sight and the gradient of the phase-front at the antenna position. It can be easily seen that if the angle of incidence is larger then the divergence angle only a small fraction of the power is coupled into the antenna. Eq. 17 with the gain function can be used under the assumption that the incident wave can be modeled by a plane wave. It can be shown that the formulas presented above for a Gaussian beam yield similiar results as eqs. (17) and (18) if the beam footprint is much larger than the antenna diameter.

\section{ITER configuration}

\subsection{The Low-Field-Side Reflectometer}

Figure 3 shows a poloidal cross-section of the ITER tokamak $\left(R_{m a}=6.2 \mathrm{~m}, a=2.0 \mathrm{~m}, B_{T}=5.3 \mathrm{~T}\right.$, $I_{P}=15 \mathrm{MA}$ ) with the proposed positions of the various reflectometer systems [4]. The LFSR has a series of waveguide transmission lines entering the vacuum vessel through an equatorial port. After a 'dogleg' (to minimize neutron steaming) the guides pass 


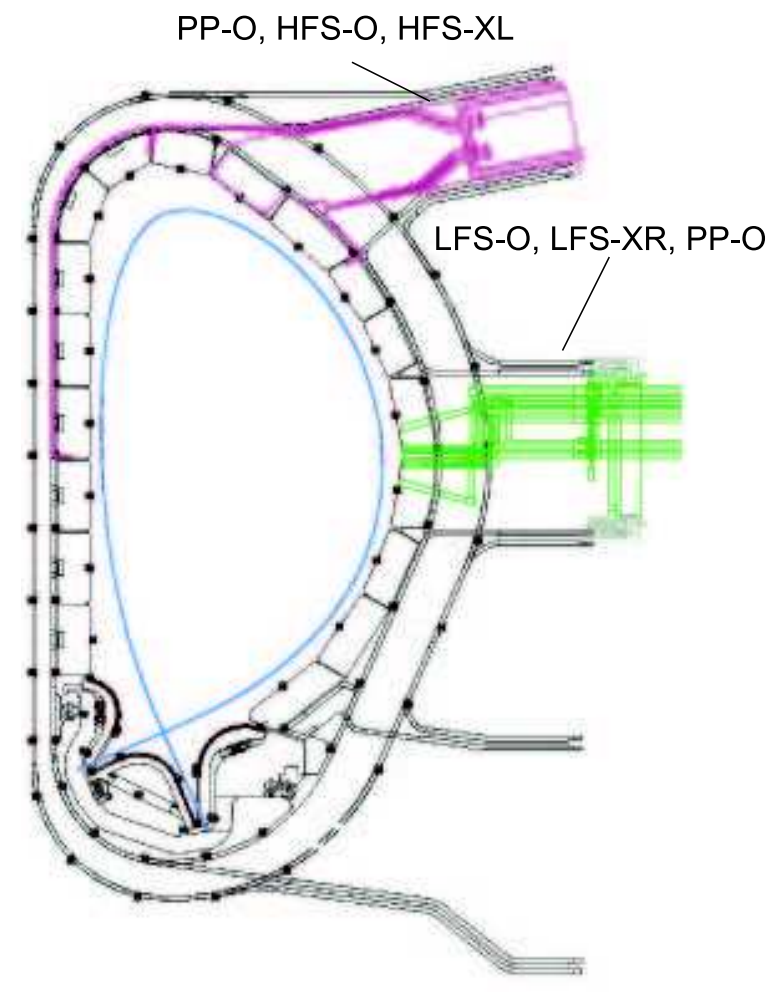

Figure 3: Poloidal cross-section of ITER showing locations of reflectometer systems: Plasma Position (PP) and High-FieldSide (HFS) systems (purple), PP and Low-Field-Side (LFS) systems (green). The LFS-O and LFS-XR systems are discussed here. (The layout is not the most up-to-date, but nevertheless illustrates the basic conditions - Figure courtesy of G.Vayakis)

through cut-outs in the shielding blanket module to face the plasma directly at the mid-plane. The antennas may terminate flush with the first wall at a radius of $R=8.498 \mathrm{~m}$, or they maybe recessed into the blanket module. The port-plug is shared with several other diagnostics and components (particularly cooling pipes) so there is some constraint on the antenna placement and alignment.

The LFSR system will operate in both O-mode and $\mathrm{X}$-mode polarization covering frequency ranges of around $18-84 \mathrm{GHz}$ (O-mode) and $120-180 \mathrm{GHz}$ (Right-hand or upper X-mode cutoff) to allow probing at full $B_{T}$ field across the ITER edge from outside the plasma last closed flux surface (the scrape-offlayer region) to as far inside the density pedestal as feasible. For half-field operation (see below) the frequency ranges are reduced to roughly $18-55 \mathrm{GHz}$ (O-mode) and $50-100 \mathrm{GHz}$ (upper X-mode cutoff) respectively.

\subsection{Equilibrium magnetic field}

The investigations reported here are based on the main ITER inductive H-mode scenario 2 regime [30]. The inset of fig. 4(a) shows a poloidal cross-section of the magnetic equilibrium for this scenario. Usually the tokamak equilibrium is considered as axisymmetric, however, the finite number of toroidal field (TF) coils (16 in the case of ITER) breaks this symmetry and leads to a small toroidal field ripple. To investigate the effect of the ripple on the propagation of the reflectometry beam requires the magnetic field of a fully three-dimensional (3D) equilibrium. With the coil currents and plasma parameters (total toroidal flux, pressure, etc.) given in [31] a 3D equilibrium was computed for one toroidal period between two coils for scenario 2 using a system of codes (VACFIELD, NEMEC, MFBE etc.). The procedure is described in $[32,33]$.

In fig. 4 the flux surfaces are plotted for the tokamak edge region (where the effect of the field ripple is strongest - and where the LFSR is intended to operate). The flux labels are the normalized poloidal flux radius $\rho_{p o l}=\sqrt{\left(\psi-\psi_{a}\right) /\left(\psi_{s}-\psi_{a}\right)}$, where $\psi_{a, s}$ are the flux values at the axis and separatrix respectively, which gives $\rho_{\text {pol }}=0$ at the axis and 1 at the separatrix. The poloidal plot shows two slices - one directly underneath a field coil and one between two coils, while the toroidal plot is at the magnetic axis height $z_{m a}=0.666 \mathrm{~m}$. The figures show that the LFS flux surfaces are compressed by approximately $1 \mathrm{~cm}$.

While the TORBEAM code can follow the beam in $3 \mathrm{D}$, it nevertheless assumes a locally axisymmetric equilibrium. However, since the change in the flux is small over the region of the beam size and displacement (see below) the effects of the field ripple can be investigated locally making (eight) poloidal slices through the three-dimensional equilibrium at different toroidal positions - indicated by the arrows in fig. 4(b). 

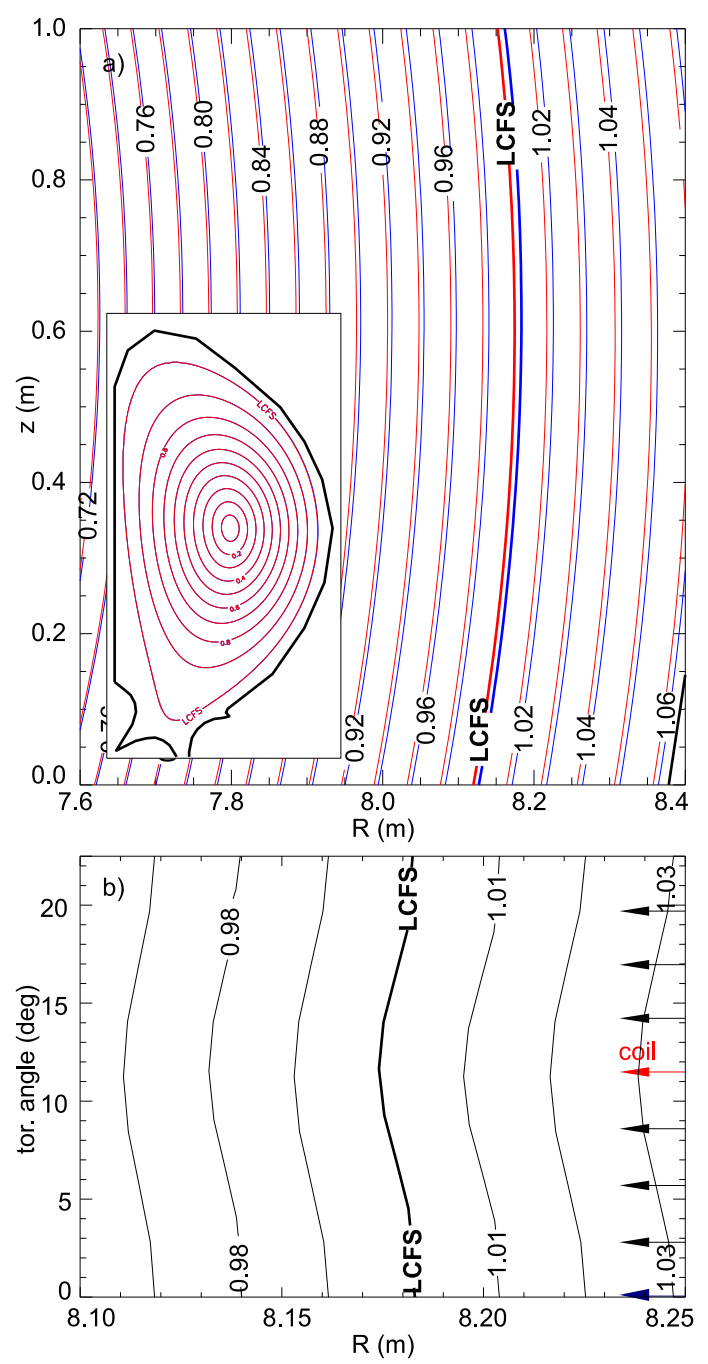

Figure 4: (a) Poloidal projection of flux surfaces at ITER midplane edge: under a TF coil (red) and between two TF coils (blue). Flux labels in $\rho_{\text {pol }}$. Inset: full poloidal cross-section of equilibrium. (b) Toroidal projection of flux surfaces at the magnetic axis height. The arrows indicate the position of the eight poloidal cuts (toroidal angles $2.8125^{\circ} \cdot n, n=0 \ldots 7$.
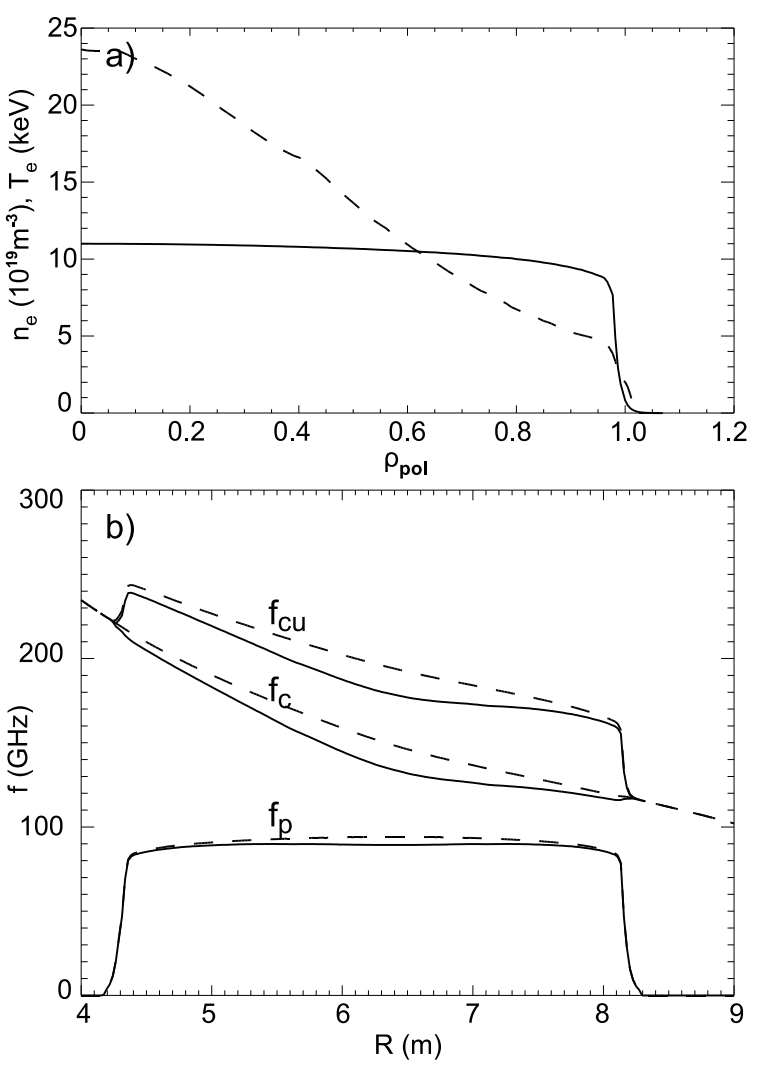

Figure 5: (a) Density (solid) and temperature (dashed) profiles as function of normalized poloidal flux radius $\rho_{\text {pol }}$ for ITER scenario 2. (b) Cutoff and cyclotron frequencies vs radius for classical (dashed) and relativistic corrected (solid): O-mode cutoff $f_{p}$, cyclotron $f_{c}$ and upper X-mode cutoff $f_{c u}$, at magnetic axis height.

\subsection{Density and temperature profile}

Figure $5(\mathrm{a})$ shows the density $n_{e}$ and electron temperature $T_{e}$ profiles as a function of $\rho_{\text {pol }}$. The profiles are based on the Polevoi profiles [19] but were modified with the addition of exponential decays to both $T_{e}$ and $n_{e}$ outside the separatrix to allow for the proper beam-tracing in, and through, the scrape-offlayer (SOL). Also, in line with recent predictions [34], the density profile is slightly peaked (peaking factor of $n_{\text {center }} / n_{\text {edge }} \approx 1.25$ ) in the core compared to the original flat profile. Note these are the same profiles employed in the work of [7] to allow for comparison. 
Figure 5(b) shows the various cutoff and cyclotron frequencies (O-mode : $f_{p}$, upper X-mode : $f_{c u}$ ) as a function of tokamak major radius at the magnetic axis height. The relativistic corrections (after Eq. 12 - solid lines) lead to a flattening of the cutoff profiles in the center of the plasma. The shallowness of the profiles, for both the $\mathrm{O}$-mode and $\mathrm{X}$-mode polarizations will make it particularly difficult for reflectometry to probe too deep inside of the pedestal, since a small variation of frequency or even small density fluctuations will shift the probing position over a very large radius. In the center even a hollow profile may occur which will preclude reflectometry completely. For the upper X-mode cutoff the core probing may be further impacted by down shifted electron cyclotron absorption from the second harmonic [4]. In addition, the longer propagation path lengths and stronger plasma curvature experienced with core probing lead to substantial beam spreading and larger beam drifting with plasma column height variations.

For these reasons, probing inside the ITER pedestal region from the LFS with reflectometry will be very challenging, and in fact will most likely to be constrained to the SOL/edge region. Thus, in this study, the LFSR antenna behaviour is principally restricted to the frequency ranges $18-84 \mathrm{GHz}$ for O-mode and $120-160 \mathrm{GHz}$ for upper X-mode. Note that in the following simulations the distance between the antenna aperture and the cutoff will always be at least equal or larger than the corresponding Rayleigh length. Hence, the modeling of the reflectometry probe beam with a Gaussian beam is always justified.

The early phases of ITER operation are likely to be performed at half toroidal field. It is expected that the LFSR should also provide reliable data during this phase. For half $B_{T}$ operation the density should decrease by a factor of $(0.5)^{4 / 3}$ while the temperature is decreased by $(0.5)^{2 / 3}$ [35]. The consequence is a decrease in probing frequency range to approximately $18-55 \mathrm{GHz}$ for O-mode and $60-95 \mathrm{GHz}$ for $\mathrm{X}$-mode. In general, the lower frequencies will lead to larger beam spreading, cf. Eq. 3 (divergence angle increases), which results in a better overlap of the reflected beam and the receiver antenna. Hence, a re- flectometer which works well at full $B_{T}$ will also work sufficiently at half $B_{T}$ operation (at least concerning coupling). The studies presented are therefore restricted to the full field scenario 2 configuration.

\section{LFSR requirements \& boundary conditions}

\subsection{Investigation criterion}

For successful operation the ITER LFSR receiver must have a sufficient signal to noise ratio $(s / n)$. The signal level will be determined by several factors: the power of the microwave source, transmission line losses, the transmission factor in the plasma $T_{p}$, and the coupling of the reflected beam back into the receiving antenna $T_{a}$. The noise level $P_{N}$ is determined partly by the detector thermal noise, but, primarily by the plasma microwave (relativistically downshifted) cyclotron and bremsstrahlung emission etc. The ECE noise power can be estimated from [18]:

$$
P_{N} \approx T_{\text {rad }} \Delta \omega / \pi
$$

where $T_{\text {rad }}$ is an effective radiation temperature and $\Delta \omega$ is the receiver bandwidth. Taking $\Delta \omega / 2 \pi=$ $1.5 \mathrm{GHz}$ and $T_{\text {rad }} \approx 10-500 \mathrm{eV}$ at the plasma edge one obtains a range for $P_{N} \approx 10^{-9}-10^{-7} \mathrm{~W}$. These are similar values to recent estimates in [37].

The transmission line losses can be minimized to a few $\mathrm{dB}$, and will affect the launch and receive signals and the noise level equally. The available source power is constrained by current technology ranging from several $\mathrm{mW}$ to few hundred $\mathrm{mW}[36,37]$ depending also on frequency. Thus the problem can be translated to one of maximizing the receive antenna $s / n=P_{r} / P_{N}=P_{o} T_{a} T_{p} / P_{N}$.

The plasma losses $T_{p}$, due to absorption (negligible) and scattering from density turbulence (small, but still to be quantified via full-wave simulations), plus the launch power $P_{o}$ and noise $P_{N}$ are generally fixed. Thus the main aim in the reflectometer front-end design is to optimize the transmission coupling factor $T_{a}$ for the full operational range of the reflectometers. For example, taking $P_{o} \sim 1 \mathrm{~mW}$, $P_{N} \sim 10^{-7} \mathrm{~W}, T_{p} \sim 1$ and $T_{a} \sim-20 \mathrm{~dB}$ would give a $s / n \sim 100$. 
The transmission coupling $T_{a}$ depends on the properties of the launch and receive antennas (gain, placement and alignment) as well as the beam propagation (refraction and diffraction). The beam propagation is strongly affected by the curvature of the plasma cutoff surfaces - which is most pronounced in the poloidal direction. This leads to a major constraint on antenna design. During the ITER main burn phase the plasma column may move vertically up and down by some $\pm 20 \mathrm{~cm}$ relative to its equilibrium height [38], while, during the current ramp-up and down phases the magnetic axis height may fall by up to $70 \mathrm{~cm}$ with strong variations in the plasma poloidal crosssection/shape. This means the probe beam will experience significant variations in the local plasma curvature leading to substantial deflections in the reflected beam (beam swinging) and consequent power variations in the receiver. Thus, in addition to maximizing $T_{a}$ it is also desirable to make the antenna configuration as insensitive as possible to beam swinging effects. How this might be achieved - at least for the main burn phase - is the subject of the current investigation. The absence of suitable magnetic equilibria for the ramp-up/down phases precludes further investigation at this stage.

\subsection{Degrees of freedom in the antenna design}

There are a number of degrees of freedom and a number of constraints on the antenna configuration.

- Antenna position: The antennas are constrained by available port space. Nevertheless there is reasonable freedom in setting the antenna height within the port-plug, as well as the radial position, i.e. the antenna apertures can be recessed in to the blanket module by some $30 \mathrm{~cm}$ or so. Recessing essentially increases the beam path length. Recessing might require some blanket material to be removed around the antenna mouth to avoid distortion of the radiation pattern, however, this should have minimal impact on the neutron flux.

- Antenna tilt: A certain amount of antenna axial tilting is possible, and, can significantly change the beam path as well as improving the receiver coupling via the antenna-beam angle of incidence. (A few degrees of tilt can be achieved via non- $90^{\circ}$ waveguide mitre-bends, but larger tilts require additional bends.)

- Antenna diameter: The diameter sets the antenna gain and affects not only the launch beam divergence but also the receiver susceptance angle. The diameter has an upper physical space constraint and a lower bound dictated, partly by manufacturing constraints but also by the $\mathrm{HE}_{11}$ waveguide mode coupling.

- Bistatic vs monostatic: In a monostatic system a single antenna both launches and receives the reflectometer beam, while a bistatic system has two separate antennas. There are advantages and disadvantages to both; for example bistatic requires twice as many antennas as monostatic, but monostatic is more susceptible to the detrimental effects of parasitic reflections in the transmission line which can severely distort the reflectometer signal [1].

- Number of antennas: Physical constraints, such as the port space, port flange area, and external vessel 'inter-space' determine the total number of antenna and waveguide feeds possible. The more antennas available the better as this increases operational flexibility. The design and layout of the port-plug is still evolving, however, current designs suggest that a maximum of 12 antennas and transmission lines is feasible.

\section{Simulation results}

In this section the basic behavior of a launched probe beam and the propagation dependence on the starting conditions of the beam, i.e. antenna diameter, position and tilting are presented. This analysis will serve as a basis for the assessment of different reflectometer options presented in the subsequent section. For the following initial analysis the $2 \mathrm{D}$ equilibrium at $0^{\circ}$ toroidal angle is used, corresponding to the position between the field coils. 


\subsection{Beam footprint and antenna diameter}

The return beam footprint is defined as the $1 / e^{2}$ intensity contour of the reflected beam at the receiver antenna plane. This can be determined directly from the numerical solution of the beam tracing equations in TORBEAM. Figure 6 shows two examples of the return footprints for a circular launched beam from a horizontal antenna at the tokamak mid-plane. The return beam cross-section has an elliptic form which can be described with major and minor widths and a rotation angle with respect to the laboratory toroidal plane. For O-mode beam polarization, fig. 6(a), the elongation is perpendicular to the magnetic field direction at the cutoff, while for the X-mode, fig. 6(b), it is along the magnetic field direction. This result was also obtained with previous ray-tracing calculations and is explained in the following references $[39,40]$.

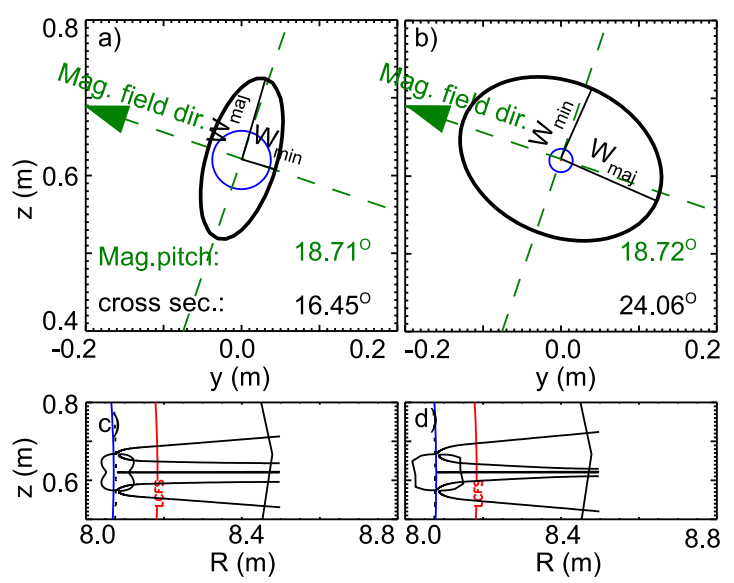

Figure 6: Return footprint of (a) $84 \mathrm{GHz}$ O-mode and (b) Xmode $160 \mathrm{GHz}$ beam. Small circle is the antenna and dashed line is the $B$ direction at the respective cutoffs. The antenna plane is flush with the blanket wall at $R=8.498 \mathrm{~m}$. (c,d) are corresponding poloidal views of the beams.

A simple explanation for the beam rotation can be found in the frame of a slab model where an analytic solution of the beam tracing equations can be obtained [18]. A Cartesian coordinate system $\left\{x_{i}\right\}$, with slab plasma confined between $-a \leq x_{1} \leq a$ and extending infinitely in $x_{2}, x_{3}$ is introduced. The strong toroidal magnetic field $B_{t o r}\left(x_{1}\right)$ is in $x_{3}$ direction and the weaker poloidal field $B_{\text {pol }}\left(x_{1}\right)$ in $x_{2}$ direction. For a beam launched at $x_{1}=a$ along $x_{1}$ (corresponding to radial propagation perpendicular to the magnetic field from the LFS) with symmetric initial beam conditions (circular cross section and isotropic phase front curvature) the rotation angle $\theta\left(x_{1}\right)$ of the beam cross-section is given by:

$$
\tan 2 \theta\left(x_{1}\right) \approx-2 \frac{\int_{a}^{x_{1}} g_{M}\left(x_{1}^{\prime}\right) \frac{B_{\text {pol }}\left(x_{1}^{\prime}\right)}{B_{\text {tor }}\left(x_{1}^{\prime}\right)} d x_{1}^{\prime}}{\int_{a}^{x_{1}} g_{M}\left(x_{1}^{\prime}\right) d x_{1}^{\prime}},
$$

where for O-mode:

$$
g_{O}\left(x_{1}\right)=X\left(x_{1}\right)\left(1-X\left(x_{1}\right)\right)^{-1 / 2}
$$

and for X-mode:

$$
\begin{aligned}
g_{X}\left(x_{1}\right)= & X\left(x_{1}\right)\left(1-X\left(x_{1}\right)\right) \\
& {\left[\left(1-X\left(x_{1}\right)\right)^{2}-Y\left(x_{1}\right)^{2}\right]^{-1 / 2} } \\
& {\left[1-X\left(x_{1}\right)-Y\left(x_{1}\right)^{2}\right]^{-1 / 2} }
\end{aligned}
$$

with $X=e^{2} n_{e} / \epsilon_{0} m_{\mathrm{eff}} \omega^{2}$ and $Y=e B_{t o r} / m_{\mathrm{eff}} \omega$. In this form the rotation of the cross section can be interpreted approximately as an average over the magnetic pitch angle $B_{\text {pol }} / B_{\text {tor }}$ with a mode dependent weighing function $g_{M}$. At the cutoff frequency for the $\mathrm{O}$-mode, i.e. the plasma frequency, the weighing function $g_{O}$ has a pole while for X-mode $g_{X}$ has poles at the cutoff and the upper hybrid resonance frequency. Thus, near the cutoff the weighing is especially strong and essentially imprints the direction of the magnetic field at this point on the footprint.

Figure 7 shows the dependence of the footprint major radius (circles) and minor radius (squares) on the launch antenna diameter $D$ for (a) O-mode and (b) X-mode polarization at three frequencies probing from the plasma SOL region: $18 \mathrm{GHz}(\mathrm{O})$ / $120 \mathrm{GHz}(\mathrm{X}) \rho_{\text {pol }} \approx 1.015$, in the middle of the pedestal: $60 \mathrm{GHz}(\mathrm{O}) / 140 \mathrm{GHz}(\mathrm{X}) \rho_{\text {pol }} \approx 0.98$, and at the top of the pedestal: $84 \mathrm{GHz}(\mathrm{O}) / 160 \mathrm{GHz}(\mathrm{X})$ $\rho_{\text {pol }} \approx 0.95$. The height of the equilibrium magnetic axis is $z_{m a}=0.666 \mathrm{~m}$ and the antenna is horizontal at a machine height of $z=0.62 \mathrm{~m}$, so that the beam 

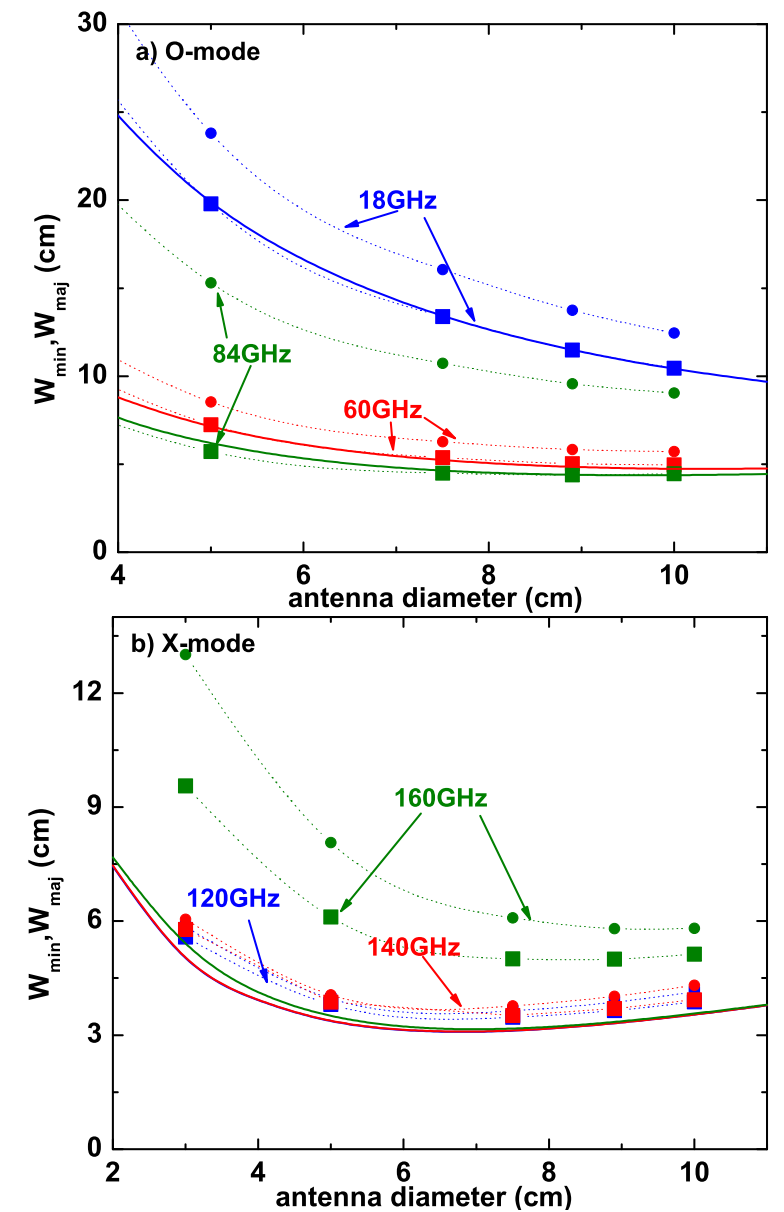

Figure 7: Return footprint major and minor widths as a function of launch antenna diameter for various frequencies in (a) O-mode and (b) X-mode. Launched antenna is at $z=0.62 \mathrm{~m}$ and $R=8.498 \mathrm{~m}$. Dashed lines are from plane mirror model.

propagates nearly purely radial. For comparison the solid lines show the equivalent circular beam radius assuming a plane mirror for the cutoff surface and propagation in vacuum. In this model the width of the circular return footprint is given by (cf. eq. (2)):

$$
w_{\text {foot }}=w_{0}\left[1+4\left(r_{\text {ant }}-r_{\text {refl }}\right)^{2} / x_{R}^{2}\right]^{1 / 2} .
$$

For O-mode the vacuum model describes quite well the minor (toroidal) width of the return footprint, while for the X-mode the model the fit is not so good, possibly due to the stronger effect of the magnetic field. At small antenna diameters the footprint size scales inversely with the diameter due to the beam divergence dominance in eq. (2) which scales as $\theta_{\text {div }} \propto$ $w_{0}^{-1} \omega^{-1}$. At larger diameters the dependence tends to linear in $D$ since the divergence plays a weaker role compared to the initial beam beam width $w_{0}$.

As a function of frequency the spot size has a minimum around the middle of the band. At low frequencies this is again due to the dominant $\theta_{\text {div }}$ scaling inversely with $\omega$, but at higher frequencies this is overtaken by longer path lengths $x$ as the beam probes deeper and a greater beam spreading due to diffraction effects and plasma curvature. For O-mode the smallest footprint is around $60 \mathrm{GHz}$ while for Xmode it is between $120-140 \mathrm{GHz}$. Likewise, radially recessing the antenna also leads to longer propagation paths and, correspondingly, to greater beam spreading etc.
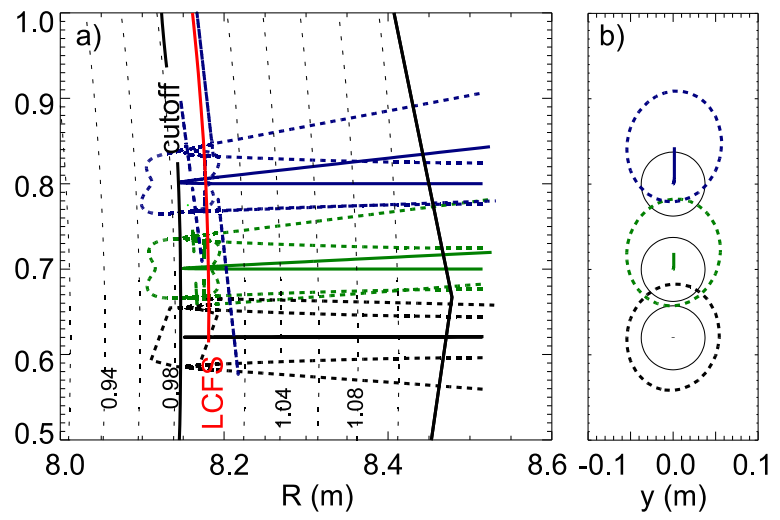

Figure 8: (a) Poloidal and (b) radial views of the propagation of $60 \mathrm{GHz} \mathrm{O}$-mode beam at different launch heights.

\subsection{Antenna position and alignment}

Generally as the beam propagates through the plasma it will experience variations in the refractive index leading to the beam drifting away from the pure vacuum path and consequently a displacement in the return beam footprint. Figure 8 shows the beam path in the poloidal plane together with corresponding radial projections of the return footprint for a $60 \mathrm{GHz}$ O-mode beam launched from three different heights. 
With increasing antenna height difference relative to the magnetic axis, the reflected beam is progressively displaced, mostly in the poloidal direction, due to the varying plasma curvature. Although the beam footprint is displaced, its size and form are almost unaffected in this case.
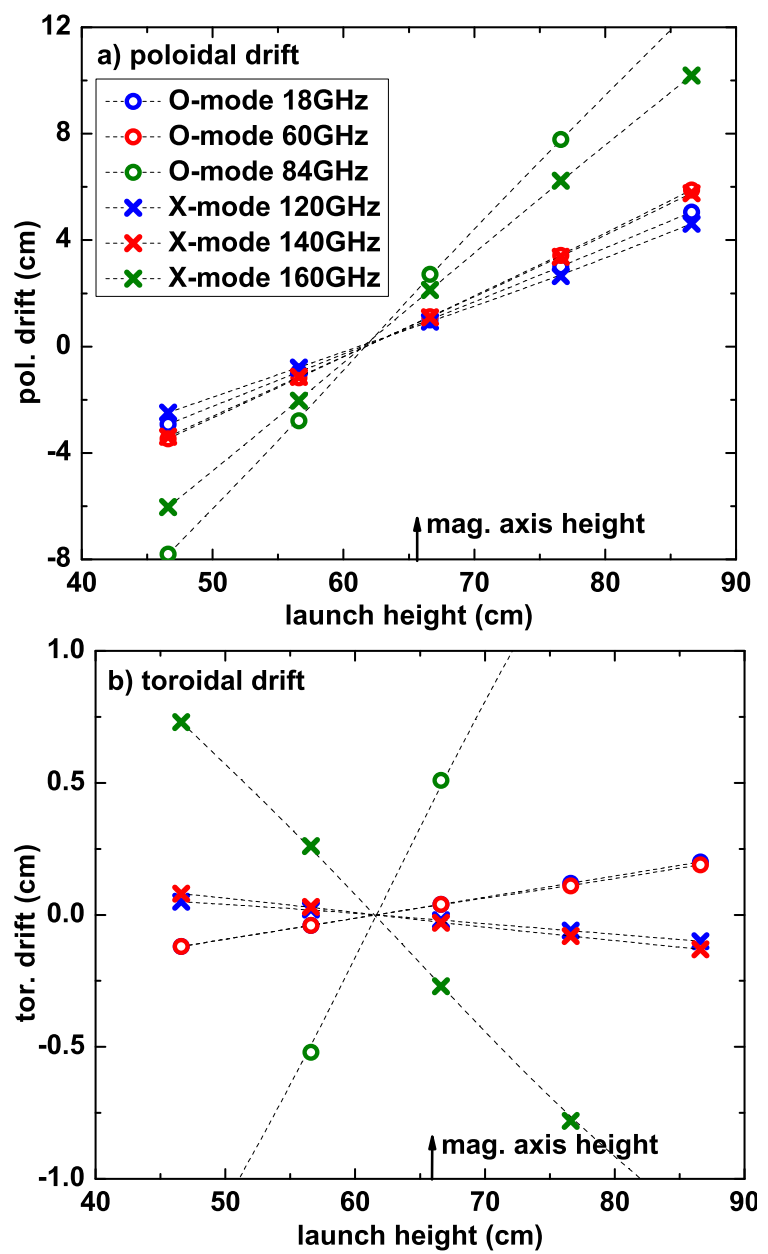

Figure 9: (a) Poloidal and (b) toroidal displacement of central ray as function of antenna launch height for $\mathrm{O}$ - and $\mathrm{X}$-mode at several frequencies. Launch and receive antenna at $R=$ $8.498 \mathrm{~m}$.

Figure 9 shows (a) the poloidal displacement and (b) the toroidal displacement of the footprint centre (i.e. the central ray) as a function of the height of a horizontally launched beam for both $\mathrm{O}$ - and X- mode with the same frequencies as in fig. 7 . The arrow marks the height of the magnetic axis at $z_{m a}=0.666 \mathrm{~m}$. Note that the curves intersect at zero displacement at $z \approx 0.62 \mathrm{~m}$, which is slightly away from $z_{m a}$ due to the magnetic equilibrium shape. All curves show roughly a linear behavior of increasing displacement with antenna height-to-magnetic-axis separation. That is, assuming non-compression of the equilibrium, the same behaviour would be seen for a vertical movement of the plasma column with the antenna fixed. As expected the toroidal displacement is significantly smaller than the poloidal, due to the corresponding radii of curvature of the flux surfaces, and, the fact that the scan is in the vertical direction. Note the toroidal displacement direction reverses between $\mathrm{O}$ - and $\mathrm{X}$-mode, as explained in $[39,40]$. The degree of beam drifting increases with the probe frequency as the beam penetrates deeper into the plasma and consequent longer path lengths.

The footprint displacements shown in figure 9 are for a horizontally launched beam. If the antenna is also tilted then there is a corresponding additional beam drift in the tilt direction. For small antenna tilts the size and shape of the return footprint is nearly unaffected.

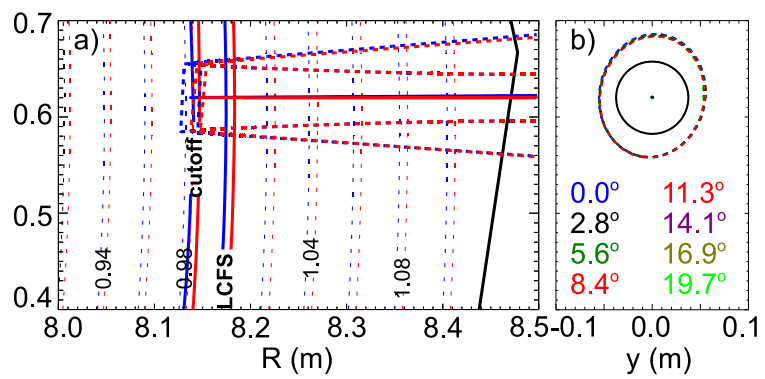

Figure 10: (a) Poloidal projection of a $60 \mathrm{GHz}$ O-mode beam propagation between two coils (blue) and at a coil (red). (b) Beam footprint (colour coded, but almost indistinguishable) from eight different toroidal cuts (numbers correspond to toroidal angle of equilibrium slice). Black circle is antenna.

\subsection{Toroidal field ripple}

In the same way that poloidal plasma curvature leads to poloidal displacement of the reflected beam, 
an enhanced toroidal curvature due to the field ripple may lead to additional toroidal displacement. The effect of varying toroidal curvature may be important with the toroidal position of the launch antenna within the port.

Figure 4 shows that the ripple is primarily a local tilting of the cutoff surface. The effect on the beam propagation is investigated using the eight toroidal cuts of the 3D magnetic equilibrium. Figure 10(a) shows an example of a $60 \mathrm{GHz}$ O-mode beam launched horizontally at the magnetic axis height for two cases: at a toroidal location between two TF coils (equivalent to the middle of an equatorial port) and a toroidal location directly beneath a TF coil. Note in each case the antennas are aligned parallel to the local major radius. At $60 \mathrm{GHz}$ the beam is reflected in the edge region where the field ripple effect is strongest. One consequence of the flux surface compression under the TF coil is that the cutoff surface is also slightly shifted inward. However, the effect of the field ripple on the propagation appears very small. Figure 10(b) shows the effect on the beam footprint for all eight toroidal cuts (colour coded) is negligible. This result was also confirmed at other frequencies, as well as for X-mode and for oblique launch angles. It should be noted however that the effect of the field ripple may be not negligible for the phase of a beam, but this is outside the scope of the current work.

\section{Assessment of reflectometer options}

Having demonstrated the behaviour of the reflectometer beam on the launch conditions we now turn to the role of the receiver antenna. A series of specific antenna scenarios are explored which cover a range of possible configurations - including poloidal and toroidal antenna alignments as well as antennas operating in monostatic or bistatic mode. In each case the power coupling efficiencies are computed for representative operational parameters, allowing a quantifiable comparison of the merits of each configuration. The assessment begins with designs where the antennas are purely horizontally aligned, then moves to the benefits of tilting, or aligning the receiver antenna with the incoming reflected beam, then finally

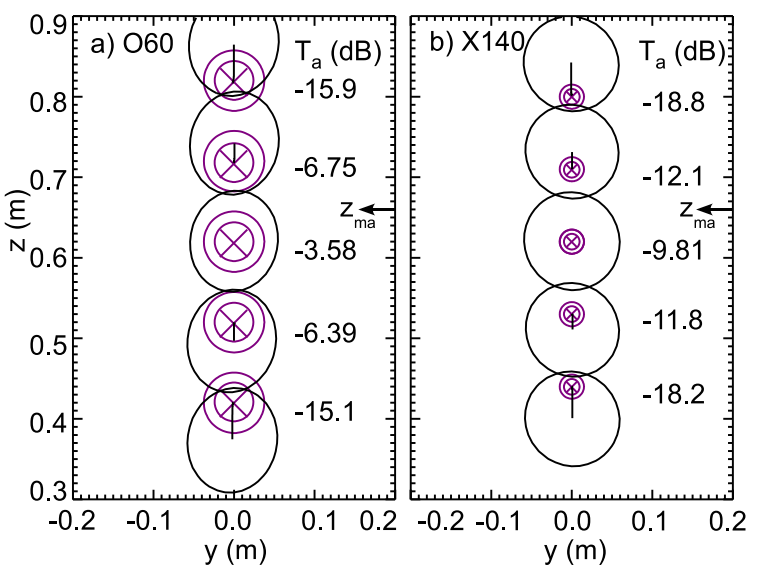

Figure 11: Poloidal monostatic antenna array with horizontal launch for (a) $60 \mathrm{GHz} \mathrm{O}$-mode and (b) $140 \mathrm{GHz} \mathrm{X}$-mode, i.e. $\rho_{\text {pol }} \approx 0.98$. Smaller circles indicate antenna and beam diameters. The coupling factor $T_{a}$ of the corresponding antenna are in $\mathrm{dB}$. $z_{m a}$ is the height of the magnetic axis.

explores the potential of hybrid configurations with multiple antennas.

\subsection{Poloidal monostatic antenna array}

Since plasma height variations create beam drifts mainly in the poloidal direction, a poloidal array of horizontally aligned antennas would seem to offer a reasonable means of capturing the return beam. To compensate for plasma height variations of $\pm 20 \mathrm{~cm}$ such an array would also need to extend roughly over this range around the mid-plane. The results from the previous section indicate that an antenna diameter of around $3 \mathrm{~cm}$ for $\mathrm{X}$-mode frequency range, and a diameter of around $7.5 \mathrm{~cm}$ for the O-mode range would give reasonable return footprint sizes, and that an antenna separation (centre to centre) of $\Delta_{z}=10 \mathrm{~cm}$ for O-mode and $9 \mathrm{~cm}$ for X-mode would require 3-5 antennas to cover the total height variation (per polarization). With the antennas mounted flush with the blanket wall $(R=8.498 \mathrm{~m})$ this configuration is similar to the design proposed in [6].

Figure 11 shows the return beam footprints and power coupling factors for (a) O-mode launched beams at $60 \mathrm{GHz}$ and (b) X-mode beams at $140 \mathrm{GHz}$ - corresponding to reflection in the middle of the 
pedestal. The beams launched from the middle antennas propagate directly back to the monostatic antenna with practically no offset and almost perfect angle of incidence. Thus the power coupling is very high at $T_{a} \sim-3.58 \mathrm{~dB}$ and $-9.81 \mathrm{~dB}$ for $\mathrm{O}$ and $\mathrm{X}$ mode respectively. Launching from antennas above or below the mid-plane leads to vertical beam drifting and to a consequent drop in coupling as the footprint overlap reduces and the angle of beam incidence increases. The X-mode coupling is somewhat poorer as the beam power is spread more over the wall rather than than just the antenna. By switching the transmission and reception from one antenna to the next as the plasma moves vertically, the poloidal monostatic antenna array offers good received signal power with good plasma height compensation ability. Because there is almost always the possibility to launch a beam close to cutoff normal, the radial coverage is rather good, including probing quite far inside the pedestal. However, as already noted, monostatic operation is susceptible to parasitic reflections and thus is less favorable.

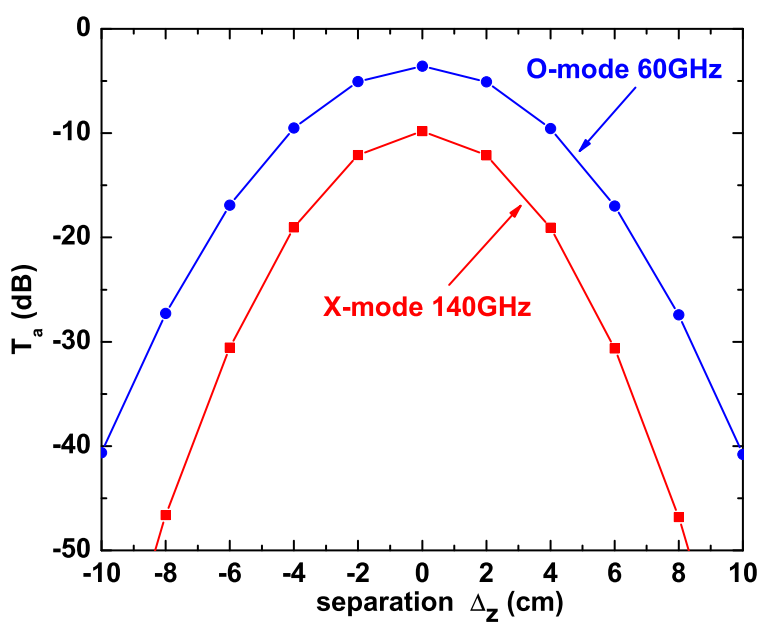

Figure 12: Coupling factor $T_{a}$ vs vertical separation of launch and receive antenna $\Delta_{z}$ for $60 \mathrm{GHz} \mathrm{O}$-mode and $140 \mathrm{GHz}$ Xmode. The launch antenna is horizontally aligned $\theta_{z}=0^{\circ}$ at $z=0.62 \mathrm{~m}$ and $R=8.498 \mathrm{~m}$. Antenna diameters are $7.5 \mathrm{~cm}$ and $3 \mathrm{~cm}$ respectively. Positive $\Delta_{z}$ values correspond to receiving above and negative to receiving below the launch antenna.

\subsection{Poloidal bistatic antenna array}

To avoid the problems of monostatic operation, the same antenna array could also be operated in bistatic, cf. $[5,7]$. Here, the beam is launched from one antenna and received on an adjacent one. However, this configuration is also not without problems. Due to the array spacing the power coupling to the adjacent antenna can be rather poor. This is shown in figure 12 with the coupling $T_{a}$ as a function of launch to receive antenna vertical separation $\Delta_{z}$ for the previous $\mathrm{O}$ and $\mathrm{X}$-mode cases with horizontally aligned antenna at $z=0.62 \mathrm{~m}$. The maximum coupling is, of course, for zero separation, i.e. monostatic operation, but drops parabolic-like with $\Delta_{z}$. For a separation of the order of the antenna diameter (minimum practical separation) the coupling is already some $-20 \mathrm{~dB}$ down. Thus, to cover the expected vertical plasma displacement would require an inordinate number of antennas.

To improve the coupling the beam return footprint size could be increased by recessing the antennas into the blanket module. Figure 13 shows a comparison of $T_{a}$ vs recess depth (zero corresponds to the wall position) for the (a) O-mode and (b) X-mode lower, middle and upper frequencies for a horizontal monostatic antenna (blue) and a horizontal poloidal bistatic pair (green) with a separation $\Delta_{z}=10 \mathrm{~cm}$. As expected, recessing the monostatic antenna reduces $T_{a}$ as the footprint expands (i.e. best close range coupling). However, for the bistatic antennas the close range coupling is very poor due to the antenna separation, but improves with recessing as the cutoff is pushed towards the antenna far-field making the configuration resemble more the monostatic. Indeed, with sufficient recessing the bistatic $T_{a}$ approaches the decaying monostatic $T_{a}$, although the curves generally will not meet due to the rotation of the elliptic footprint. Both the $\mathrm{O}$ and $\mathrm{X}$-mode coupling values and behaviour closely match coupling simulations using vacuum Gaussian beams [41].

Recessing by $0.3 \mathrm{~m}$ (to $R=8.798 \mathrm{~m}$ ) raises the coupling sufficiently such that 5 antennas could perhaps reasonably cover the plasma displacement range. An example of this is shown in fig. 14 for (a) launching from the middle antenna and (b) from the second 

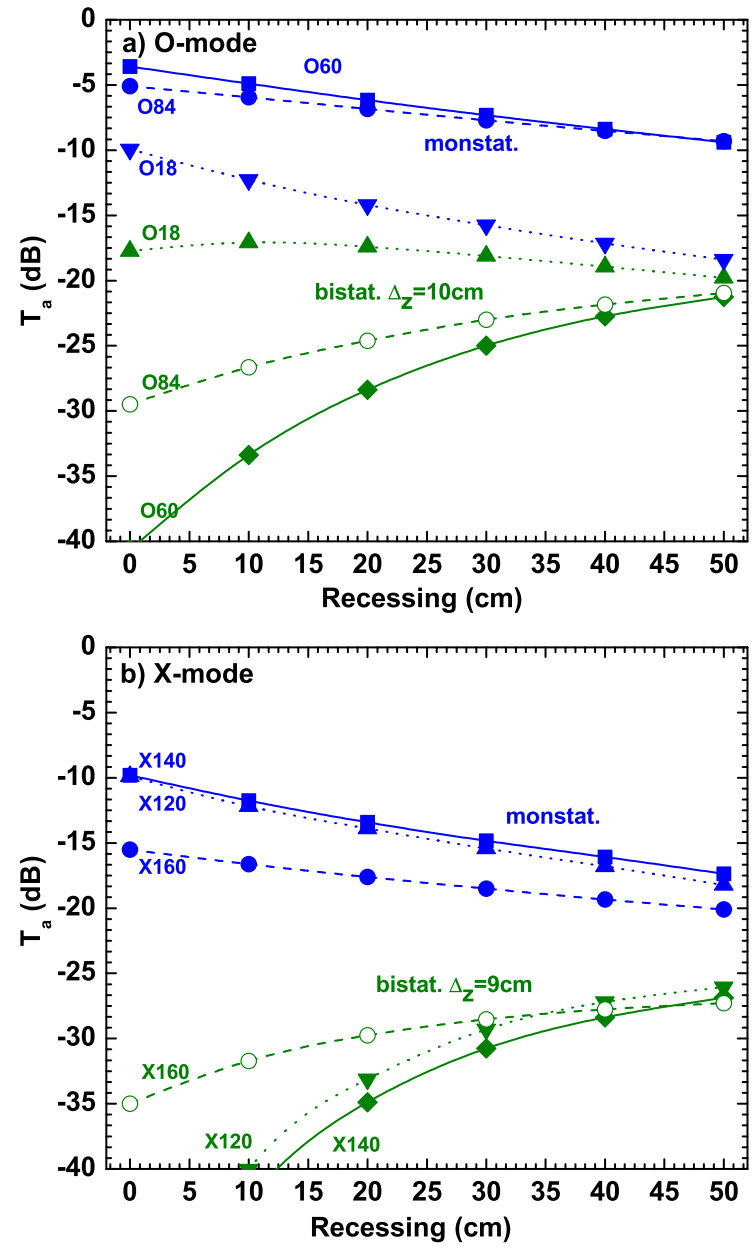

Figure 13: Coupling factor $T_{a}$ vs recess depth for horizontal monostatic antenna (blue) and horizontal poloidal bistatic antenna pair $\theta_{z}=0^{\circ}$ (green) with $\Delta_{z}=10 \mathrm{~cm}$ for (a) O-mode $18 \mathrm{GHz}$ (dotted), $60 \mathrm{GHz}$ (solid), $84 \mathrm{GHz}$ (dashed), and (b) Xmode $120 \mathrm{GHz}$ (dotted), $140 \mathrm{GHz}$ (solid), $160 \mathrm{GHz}$ (dashed).

upper antenna. The return footprint is in good agreement with that shown in fig. 3a of [7] where the simulation was performed with ray-tracing. Although there is an adequate footprint overlap with the neighboring antennas the power coupling is still at best only $-25.0 \mathrm{~dB}$ due to the larger beam spreading, and due to beam phase-front being poorly aligned with the receiver antenna. For the upper launch case, fig. 14(b), the overlap with the uppermost antenna

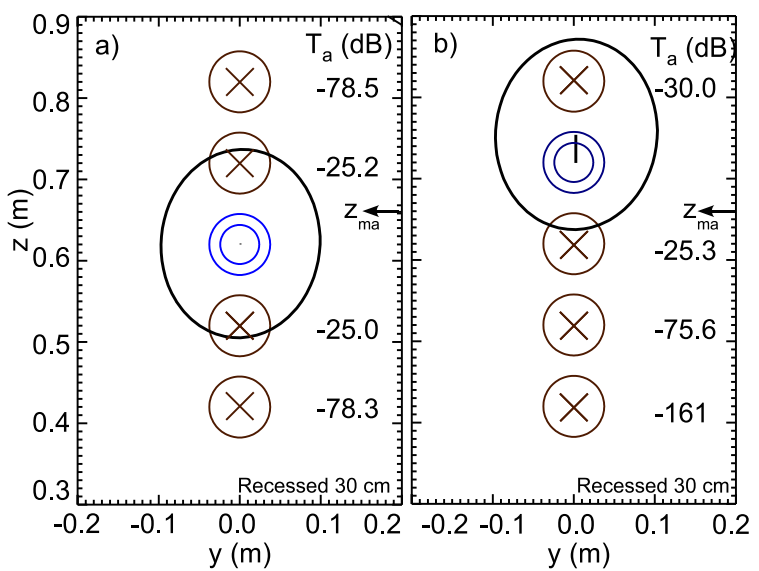

Figure 14: Poloidal bistatic horizontal aligned array. Antennas separated by $\Delta_{z}=10 \mathrm{~cm}$ and recessed by $0.3 \mathrm{~m}$ to $R=8.798 \mathrm{~m}$. Blue circle is the launch antenna and brown circles with crosses are receiver antennas. O-mode $60 \mathrm{GHz}$ launch from (a) middle antenna and (b) second upper antenna.

is much better due to the poloidal displacement, but also here the coupling is only $-30.0 \mathrm{~dB}$, while the coupling to the middle antenna is higher at $-25.3 \mathrm{~dB}$ although there is a poorer footprint overlap. The reason is that the phase-front of the reflected beam is actually better aligned with the middle antenna than with the uppermost antenna. Essentially, for this particular geometry the variation in the plasma curvature with height is small such that reversing the launch and receive antenna positions plays no role and antenna reciprocality holds. A recessed poloidal bistatic array could thus in principle compensate the plasma height variations reasonably well, but at the cost of reduced receive signal power compared to a pure monostatic array.

\subsection{Bistatic pair with tilted launch antenna}

The discussion above indicates that the receiver antenna should be aligned to the phase-front of the reflected beam to achieve maximum power coupling. That is to say, for bistatic operation an antenna tilt should be introduced to match the reflected beam incidence (optimal phase-front) to the maximum in the receiver antenna radiation pattern (directionality). 


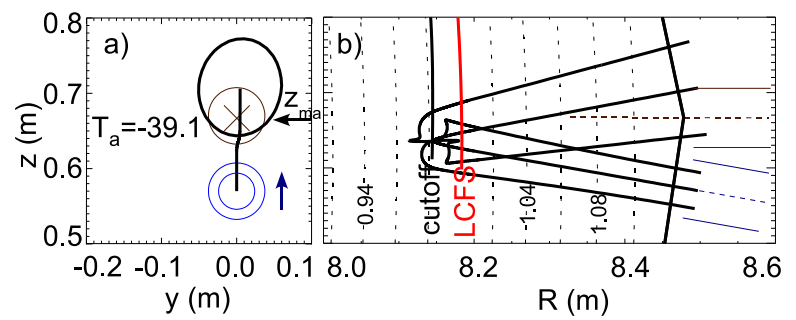

Figure 15: (a) Radial and (b) poloidal views of $\Delta_{z}=10 \mathrm{~cm}$ separated bistatic pair with horizontal receive and $\theta_{z}=10^{\circ}$ upward tiled (arrow) launch antennas for $60 \mathrm{GHz}$ O-mode.

First, a poloidal bistatic antenna pair is considered with a launch antenna poloidally tilted $\theta_{z}=10^{\circ}$ towards the receive antenna. The receive antenna remains horizontally aligned. Although the footprint overlap, as shown in the example in fig. 15, can be good, the coupling remains poor due to the phasefront mismatch. Thus, for bistatic operation it appears necessary to tilt, i.e. align, both the launch and receive antennas.

\subsection{Poloidal tilted bistatic antenna pair}

Figure 16 shows the return footprint behaviour with both antennas tilted towards each other (lower antenna launch) for the usual O- and X-mode frequencies at various plasma heights. Plasma height variations were simulated by shifting the antenna with respect to the equilibrium.

Tilting means the antenna vacuum lines-of-sight are no longer parallel but cross at some distance, set by the antenna separation and tilt angles, creating an optimal measurement range. For both non-recessed antenna cases in fig. 16 with $\Delta_{z}=10 \mathrm{~cm}$ (O-mode) and $9 \mathrm{~cm}$ separation (X-mode), the optimal tilt angle was found to be $\theta_{z}= \pm 5^{\circ}$, relative to the horizontal (i.e. $10^{\circ}$ subtended angle). For the nominal plasma height (solid black) the coupling is $-11.5 \mathrm{~dB}$ to $-7.88 \mathrm{~dB}$ for O-mode, and $-16.8 \mathrm{~dB}$ to $-13.6 \mathrm{~dB}$ for X-mode.

Interchanging the transmit and receive antenna positions has a small $(2-3 \mathrm{~dB})$ effect, but only at high frequencies and for large plasma height displacement.

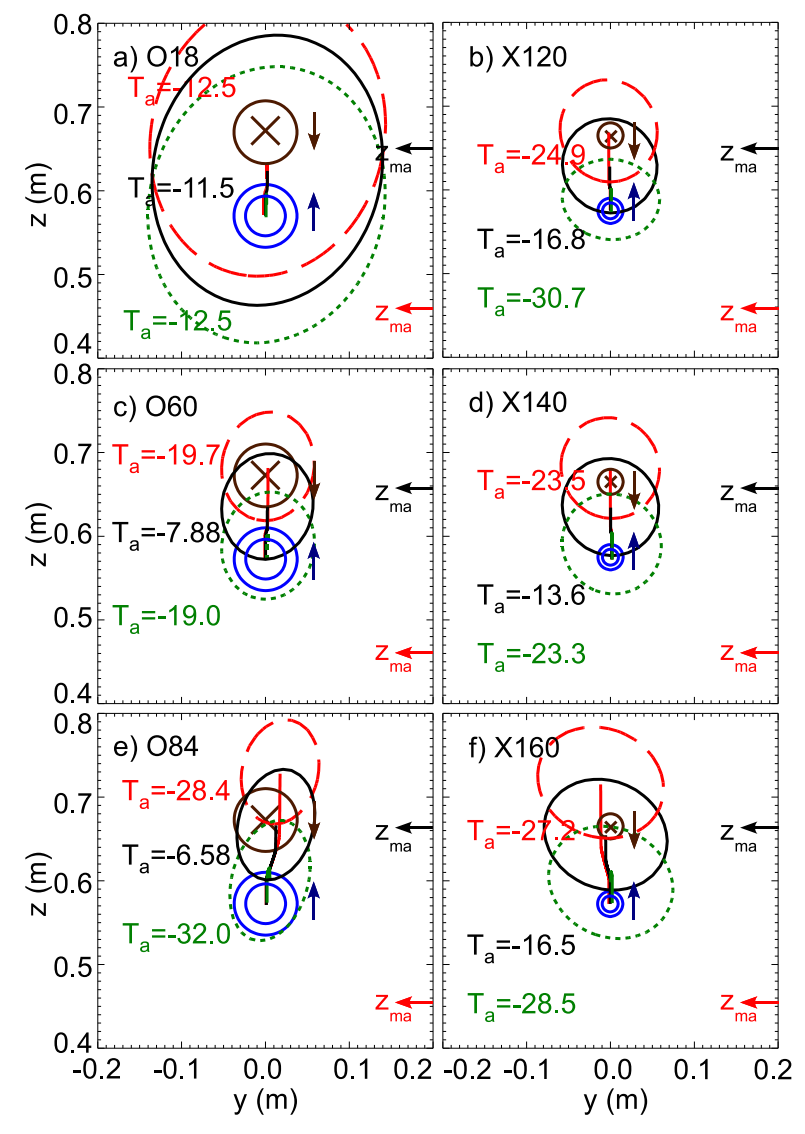

Figure 16: Poloidal tilted bistatic pairs $\left(\theta_{z}= \pm 5^{\circ}, \theta_{t}=\right.$ $0^{\circ}$ ) at various plasma heights: Nominal plasma $z_{m a}$ (black solid), $20 \mathrm{~cm}$ lower (red dashed), and $20 \mathrm{~cm}$ higher (green dotted). Left column: $18-84 \mathrm{GHz}$ O-mode, right column: $120-160 \mathrm{GHz}$ X-mode.

This is due principally to the stronger plasma curvature for deeper high frequency probing which breaks the symmetry of the reflection.

\subsection{Toroidal tilted bistatic antenna pair}

For a poloidally separated bistatic antenna pair with poloidal tilting the beam drifts due to the tilting and the plasma height variation are coupled and can add or subtract from each other. However, if the antenna separation is aligned in the toroidal direction, then essentially the plasma curvature and antenna 

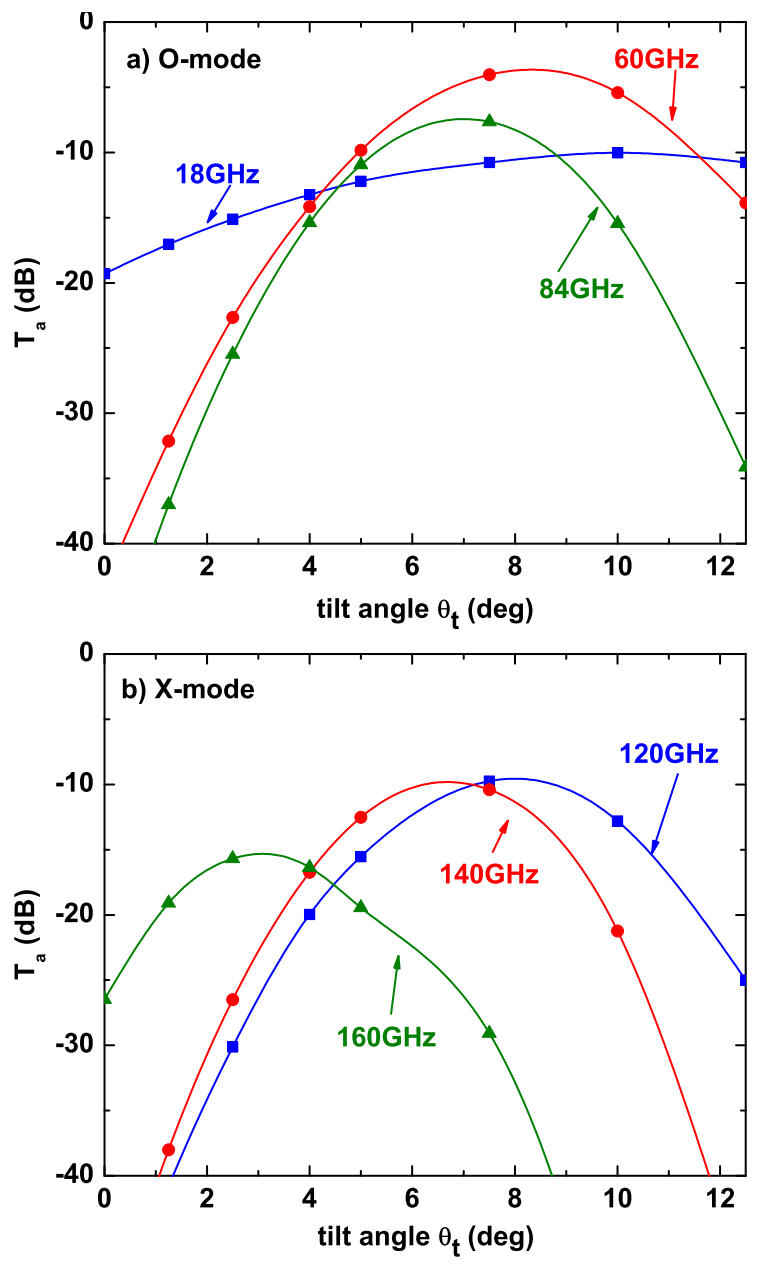

Figure 17: (a) O-mode and (b) X-mode received power coupling vs tilt angle for toroidal tilted bistatic antenna pair.

tilt effects can be separated - and individually compensated.

For a toroidal tilted bistatic pair figure 17 shows the power coupling $T_{a}$ vs tilt angle $\left(\theta_{t}\right.$ in the toroidal direction relative to the radial normal - the subtended angle is double) for the usual antenna parameters: (a) O-mode with $7.5 \mathrm{~cm}$ diameter and $\Delta_{t}=10 \mathrm{~cm}$ separation, and (b) X-mode with $3 \mathrm{~cm}$ diameter and $9 \mathrm{~cm}$ separation. The antenna height is again $z=$ $0.62 \mathrm{~m}$. At zero tilt the antennas are parallel to each other and the received power coupling is poor as the beam returns directly back to the launch antenna and misses the receiver. With increasing tilt angle the reflected beam shifts towards the receiver and the power coupling rises to a maximum before falling once the beam passes the receiver. The optimum tilt depends on the frequency. With increasing frequency the optimal tilt falls as the propagation path becomes longer. For O-mode the optimum tilt value is around $\theta_{t}= \pm 7.5^{\circ}$, where the 60 and $84 \mathrm{GHz}$ cases reach their maximum. For the $18 \mathrm{GHz}$ case (SOL probing) the received power remains nearly constant in this region.
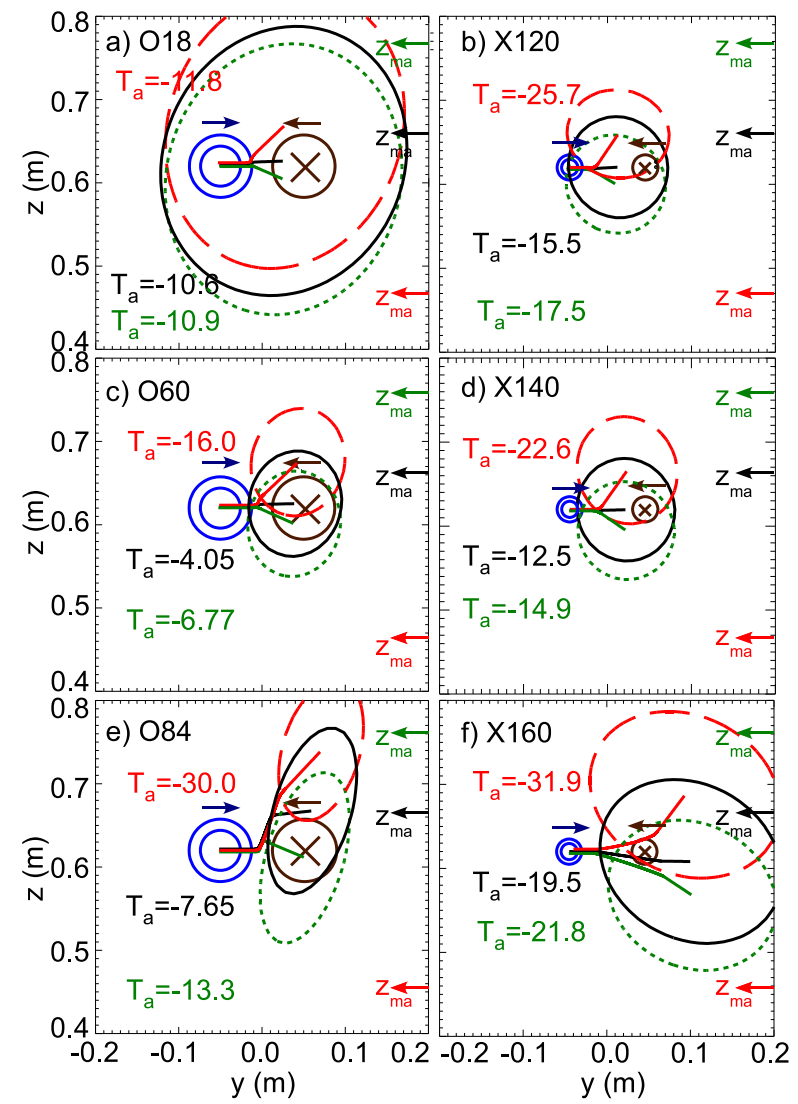

Figure 18: Toroidal tilted O-mode: $\theta_{z}=0^{\circ}, \theta_{t}= \pm 7.5^{\circ}$, $\Delta_{t}=10 \mathrm{~cm}$ and X-mode: $\theta_{z}=0^{\circ}, \theta_{t}= \pm 5^{\circ}, \Delta_{t}=9 \mathrm{~cm}$ bistatic pairs at various plasma heights: Black solid: nominal plasma $z_{m a}$, red dashed: plasma $20 \mathrm{~cm}$ lower, and dotted: plasma $+10 \mathrm{~cm}$ higher. Left column: $18-84 \mathrm{GHz}$ O-mode, right column: $120-160 \mathrm{GHz} \mathrm{X}$-mode. 
For X-mode the optimum tilt angle for 120 and $140 \mathrm{GHz}$ is again around $\theta_{t}=7^{\circ}-8^{\circ}$. But with such a tilt nearly no signal power would be received at $160 \mathrm{GHz}$. Thus a value of $\theta_{t}= \pm 5^{\circ}$ would appear to be a good compromise. The optimum tilt angle is of course also dependent on the antenna separation - the smaller the separation, the smaller the optimum tilt angle and the larger the antenna line-of-sight crossing distance.

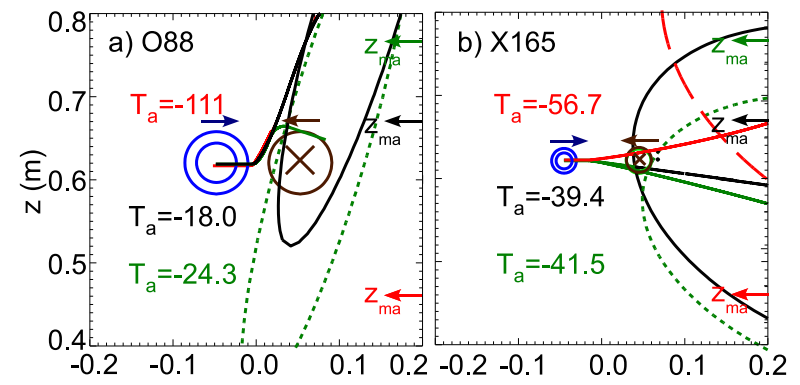

Figure 19: Same as fig. 18 for operation inside pedestal. (a) O-mode at $88 \mathrm{GHz}, \rho_{\text {pol }} \approx 0.84$. (b) X-mode at $165 \mathrm{GHz}$, $\rho_{\text {pol }} \approx 0.86$.

The behaviour of the beam footprints for the toroidal bistatic configuration is shown in fig. 18 for various plasma heights. For a plasma height at its nominal equilibrium value (solid black) the power coupling is $-10.6 \mathrm{~dB}$ to $-4.05 \mathrm{~dB}$ in O-mode, and $-19.5 \mathrm{~dB}$ to $-12.5 \mathrm{~dB}$ in $\mathrm{X}$-mode. These values are comparable to the poloidal monostatic array. For plasma height variations of $10 \mathrm{~cm}$ (green) and $20 \mathrm{~cm}$ (red) there arise notable poloidal displacements. At low frequencies, moderate plasma height variations can be compensated easily, but at high frequencies and large plasma height variations the displacements become rather large and the coupling drops to below $-30 \mathrm{~dB}$

At even higher probe frequencies, $88 \mathrm{GHz}$ O-mode and $165 \mathrm{GHz} \mathrm{X}$-mode, fig. 19, corresponding to cutoffs well inside the pedestal, $\rho_{\text {pol }} \approx 0.85$, there is still a reasonable height coverage. In general the beam drifts are now quite large, but also the size of the return footprint increases so that, at least for the O-mode, at moderate plasma height variations the coupling is moderate. But very large plasma height variations can not be compensated. For the X-mode, reflectometry inside the pedestal with this configuration will be very difficult.

In general the toroidal bistatic coupling values are rather similar to those of the poloidal bistatic antenna pair, except that the sensitivity to plasma height variations tends to be somewhat better.

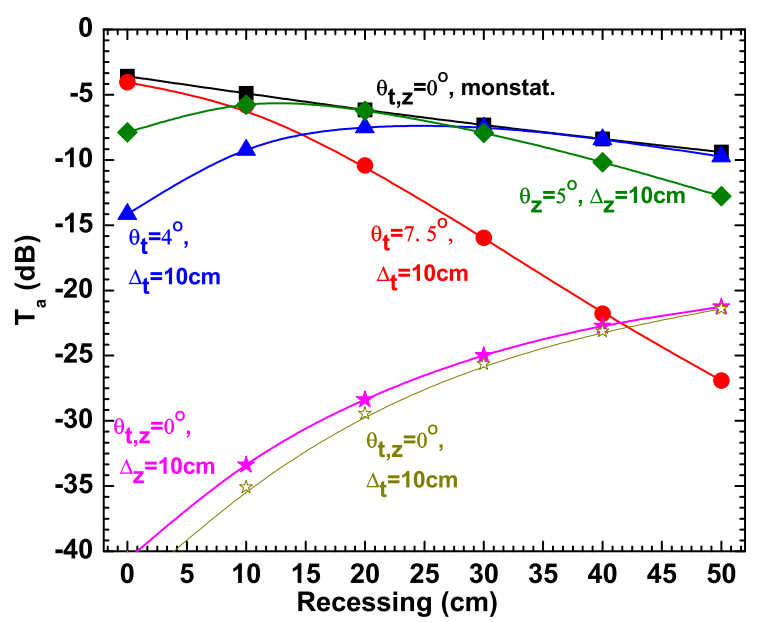

Figure 20: Coupling factor $T_{a}$ vs recess depth for $60 \mathrm{GHz}$ O-mode. $\Delta$ is the antenna separation and $\theta_{t, z}$ the antenna tilt angle. Monostatic (Black squares), tilted toroidal bistatic $\theta_{t}= \pm 7.5^{\circ}$ (red circles) and $\theta_{t}= \pm 4^{\circ}$ (blue triangles), tilted poloidal bistatic $\theta_{z}= \pm 5^{\circ}$ (green hashes), non-tilted poloidal bistatic $\theta_{t, z}=0^{\circ}$ (magenta filled stars), and non-tilted toroidal bistatic $\theta_{t, z}=0^{\circ}$ (dark yellow hollow stars).

The effect of radially recessing the antennas was also investigated for the tilted bistatic case. Figure 20 compares the coupling $T_{a}$ vs recess depth for various configurations with $60 \mathrm{GHz} \mathrm{O}$-mode probing. The toroidal bistatic case of fig. $18(\mathrm{c})$ with $\Delta_{t}=10 \mathrm{~cm}$ and $\theta_{t}= \pm 7.5^{\circ}$ case (red circle symbols), which is optimized for no recessing, follows a similar trend as the monostatic case (black squares), but has a sharper decay due to the additional effect of increasing beam displacement as well as the growing footprint with longer propagation paths. However, reducing the tilt to $\theta_{t}= \pm 4.0^{\circ}$ (blue triangles) better optimizes the configuration for recessing. The reduced tilt moves the optimal measurement range outward increasing the longer range $T_{a}$ (i.e. deeper recess) but at the 
expense of closer range coupling. For comparison the equivalent poloidal tilted bistatic case $\Delta_{z}=10 \mathrm{~cm}$ and $\theta_{z}= \pm 5^{\circ}$ case (green diamonds) is slightly better optimized for a smaller recess, while the two nontilted cases $\theta_{t}=0^{\circ}$ (dark yellow stars) and $\theta_{z}=0^{\circ}$ (magenta stars) are equally poor. Again, the frequency dependence of the bistatic tilted $T_{a}$ with recess is similar to the vacuum Gaussian beam predictions [41].

Although antenna recessing enhances the beam drifting effect with plasma height variations, the correspondingly larger return footprints gives better overlap with the receiver antenna, resulting in rough cancellation of effects. Essentially, recessing does not appear to have a particularly strong impact on the sensitivity to plasma height variations.

\subsection{Monostatic hybrid system}

The simple toroidal bistatic antenna pair considered so far offers adequate performance, except perhaps at high frequencies and large plasma height displacements. Here, a degree of optimization is possible, for example reducing the antenna separations and tilt angle, which could improve the radial coverage inside the pedestal region. However, an alternative approach is to combine a monostatic configuration with a bistatic system so as to unite the advantages of each and diminish the disadvantages of both. Such antenna configurations are broadly labeled hybrid systems.

One such configuration was already considered in fig. 15 where the receive antenna of a poloidal bistatic pair remained horizontal. Here, the receive antenna could also be used in monostatic mode. The receive power coupling in fig. 15 was found to be rather poor; however, it might be sufficiently improved by reducing the antenna separation to the technical minimum (antennas almost touching). Such a configuration could be classed as a monostatic hybrid. The ability to use a bistatic system in monostatic mode is actually rather important from an operational viewpoint. If one antenna, or transmission line is damaged, or becomes inoperable then it is still possible to use one half of the pair as a monostatic antenna. This is a strong argument for generally reducing the antenna spacing in any bistatic pair.

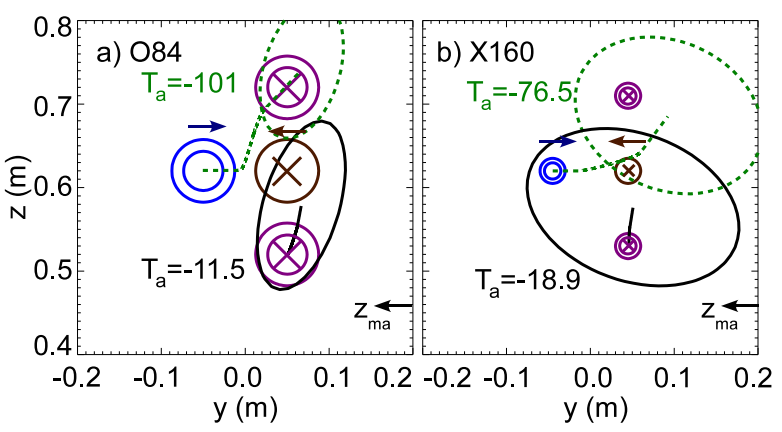

Figure 21: Bistatic hybrid system with toroidal tilted bistatic pair $\left(\theta_{t}= \pm 7.5^{\circ}\right.$, see arrows $)$ and two poloidally displaced non-tilted $\left(\theta_{t, z}=0^{\circ}\right)$ monostatic antennas. Footprints are for a plasma $20 \mathrm{~cm}$ below nominal height, (a) $84 \mathrm{GHz} \mathrm{O}$-mode and (b) $160 \mathrm{GHz}$ X-mode. Two cases: Launch from tilted (blue) antenna and receive at upper monostatic (dotted green); launch and receive from lower monostatic horizontally aligned antenna (solid black).

\subsection{Bistatic hybrid system}

To a toroidal bistatic pair more horizontal aligned receive/monostatic antennas can be added. Figure 21 shows an example with additional horizontal antennas above and below the usual tilted bistatic receiver antenna: (a) $\Delta_{z}=10 \mathrm{~cm}$ for O-mode and (b) $9 \mathrm{~cm}$ for $\mathrm{X}$-mode. The cases shown are for the difficult or extreme operation at high frequency and large plasma height displacement. This configuration offers the opportunity to either launch from the tilted antenna and receive at the upper antenna (dashed green), but here the receive coupling is very small due to phasefront mismatch to the horizontal monostatic antenna; or to launch and receive from the lower monostatic antenna. As may be expected the coupling for both O- and X-mode is comparatively high. With such a system the whole edge region can be covered rather well. In critical cases, i.e. large plasma height variations and high frequencies, the monostatic antennas can be used.

\subsection{Tristatic cluster}

Continuing the theme of multiple antennas, fig. 22 shows an example of a poloidal array of receive antennas with a toroidally displaced launch antenna. The difference here is that the receive antennas are 


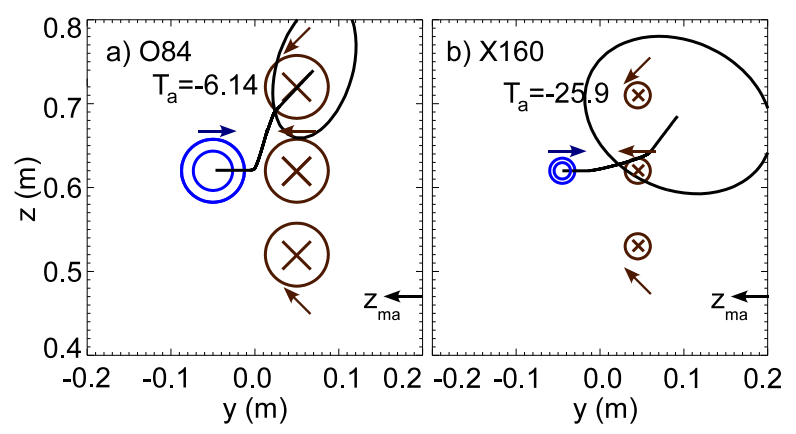

Figure 22: Tristatic cluster with optimally tilted (see arrows) poloidal array of receivers. Plasma is $20 \mathrm{~cm}$ below nominal; (a) O-mode $84 \mathrm{GHz}$, upper and lower antenna: $\theta_{t}=7.5^{\circ}, \theta_{z}=$ $\pm 7.5^{\circ}$, middle antennas: $\theta_{t}= \pm 7.5^{\circ}, \theta_{z}=0^{\circ}$ ) (b) X-mode $160 \mathrm{GHz}$, upper and lower antenna: $\theta_{t}=5^{\circ}, \theta_{z}= \pm 5^{\circ}$, middle antennas: $\theta_{t}= \pm 5^{\circ}, \theta_{z}=0^{\circ}$ )

all tilted towards the launch antenna - poloidally and toroidally - as indicated by the arrows. This configuration essentially combines the benefits of the toroidal bistatic pair with an aligned poloidal bistatic array. The extreme operational cases are again shown. Contrasted to the previous bistatic/monostatic hybrid of fig. 21 the receive coupling is significantly improved and the system now offers full coverage over the edge region even with large plasma vertical displacements. Nevertheless, deep access inside the pedestal is still limited due to large drifts arising from the tilts, but the problem of parasitic reflections arising in monostatic operation is avoided.

If monostatic is allowable, then comparable performance could be obtained with three antennas by combining the launch antenna with the centre receive antenna, which would be horizontal, with the two outer receive antennas still tilted towards the central launch.

\subsection{Design comparison}

Several antenna configurations have been presented and their performance analyzed. Each configuration can be further optimized with a delicate adjustment of the various parameters. More exotic and sophisticated designs can also be devised, although mostly at the cost of additional antennas. However,

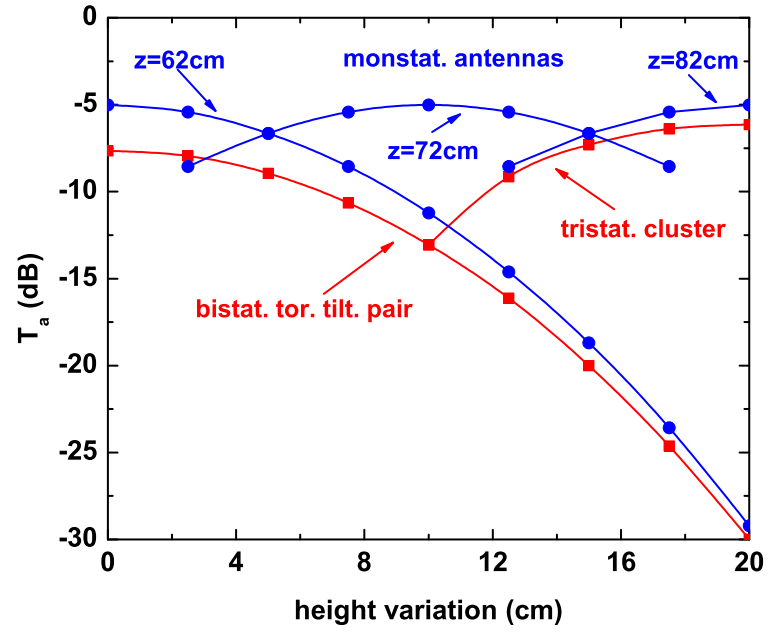

Figure 23: Coupling vs plasma height for various configurations with $\mathrm{O}$-mode $84 \mathrm{GHz}$. Monostatic horizontal antenna array at indicated heights (blue circles). Toroidal tilted bistatic pair (red squares), upper branch is for tristatic cluster.

the basic influence (and interaction) of the main antenna parameters, such as position, tilt, monostatic or bistatic, and separation, on the performance of the main configurations can be identified and summarized.

The poloidal monostatic antenna array has in general the highest coupling factor $T_{a}$. But if monostatic operation is not acceptable then operation in bistatic mode requires tilting of (preferably both) the launch and receive antennas. For maximal receive power coupling the receiver antenna(s) should be tilted towards the launch antenna. However, launch antenna tilting introduces additional frequency dependent beam drifts - which means that good optimization can usually only be achieved for a particular frequency band. Bistatic antenna tilting also affects the measurement depth-of-field - the radial region over which good coupling is achieved. Large tilt angles improve the coupling at close range, but at the expense of long range coupling. Generally, the smaller the bistatic antenna seperation the smaller the correspondingly tilt angles (and thus tilt induced beam drifts) - which reduces the frequency dependency and increases the long-range coupling depth. Reducing the tilt dependent beam drifts also allows 
a bistatic system to operate quite well in monostatic mode should one half of the antenna pair fail.

Plasma vertical movements have a particularly strong effect on the return beam at high probe frequencies. To compensate plasma height variations a poloidal arrangement of antennas is recommendable. In fig. 23 the coupling factor $T_{a}$ is plotted for $84 \mathrm{GHz}$ O-mode probing vs the height difference between the antenna and the plasma magnetic axis for several antenna configurations. Excellent overall coupling can be achieved with the poloidal monostatic array, but the transceiver must be switched from one antenna to another as the plasma moves. The toroidal bistatic antenna pair has similar coupling behaviour vs height variation as a single monostatic horizontally aligned antenna. As with the monostatic array, adding further bistatic pairs or extra single receiver antennas (hybrid designs, tristatic cluster) can extend the good coupling range.

With antenna arrays, or multiple receive antennas, there is now the requirement to either instrument each line, i.e. multiple reflectometer systems on each antenna, or some form of real-time waveguide switching. Waveguide switching can lead to signal interuption during switching, plus the need for realtime control and sophisticated switching decision criterion during fast transients. In either case there is additional component/system expense.

In general, height compensation is a compromise between using many antennas - at the cost of dynamic antenna switching - or using a lower antenna gain (diameter) to increase the return footprint - but at the expense of power coupling, particularly for deep plasma probing / high frequencies. Reduced power coupling on the other hand may be offset by inceasing the launch power or the sensitivity of the receiver detectors.

For a fixed antenna gain the return footprint can be enlarged by recessing the antennas radialy. For a fixed bisatic antenna separation the tilt angles should be reduced to retain optimal coupling with increasing recess depth. The corresponding larger beam drifts with plasma height variations compete with the larger return footprint resulting in a coupling that is nearly independent of recess depth.

Antenna recessing can also have a technical impact.
It will be necessary to remove some of the wall material around the antenna mouth to avoid distortion of the beam pattern, which may reduce the overall neutron shielding in the port plug, however, a conical recess (cutout) can reduce multi-path reflections. Recessing may also help in cooling of the antenna system.

The question of which configuration is superior is ultimately somewhat subjective and more a question of cost, tolerance to risk, and the specific measurement need. These last two topics have not been touched on in this analysis, which has concentrated more on performance issues.

\section{Summary}

The behaviour of a microwave reflectometer diagnostic beam, from the launch antenna, through the plasma and back to the receive antenna, has been modeled with the aid of beam-tracing and Gaussian beam coupling equations. The technique extends previous simulation approaches using ray-tracing to provide a more realistic and quantifiable measure of the transmitter to receiver power flow. Specifically, the method fully incorporates the influence of both the beam intensity at the receiver and the alignment of its phase-front with the antenna radiation pattern.

Applying the technique to the ITER geometry using a 3D magnetic equilibrium of the scenario-2 $\mathrm{H}$ mode with the TORBEAM code, the effect of toroidal field ripple on the propagation of a reflectometer beam launched from the tokamak LFS was found to be negligible in ITER. For the ITER scenario-2 equilibrium with a slightly peaked density profile, the reflectometer beam sensitivity on the launch antenna properties and the plasma shape (vertical plasma displacement) were investigated. The behaviour of several possible LFSR antenna configurations were then assessed and their relative merits contrasted. For bistatic operation it was found that, in general, the receive antenna should be tilted to match the phasefront of the incoming beam. A poloidal or toroidal tilted bistatic antenna pair appears to offer a robust (and cheap) solution for a reflectometer intending to diagnose the SOL/edge density profile. The problems 
of beam displacement at large plasma height displacements might be solved with additional antennas, as presented by hybrid systems and the tristatic cluster.

The beam-tracing/power-coupling method is well suited for a comparative study of different reflectometer antenna systems applied to identical plasma scenarios and geometries. However, to provide an absolute value of the received power, or the $s / n$, the simulation would need to include more precise estimates of the plasma noise emission, as well as incorporating the effects of beam power losses due to (turbulence) scattering and non-resonant absorption.

A more detailed study of the reflectometer sensitivity to a range of other equilibria, density and temperature profiles (e.g. other peaking factors), as well as the half field operation and the behaviour of the reflectometer phase signals are left for future work.

\section{Acknowledgments}

The authors thank R.Bilato, U.Mszanowski, F.Volpe and R.Zille for contributions to the TORBEAM code development; G.Wang, W.A.Peebles and T.Rhodes for sharing early preprints of their work plus density and temperature profiles, and G.Vayakis for fig.3. G.R.Hanson is gratefully acknowledged for fruitful discussions. This work was performed as part of a diploma thesis at the Technische Universität München.

\section{Appendix A. Supplement to coupling}

Equation 15 is the general formula for the power coupling of asymmetric beams (elliptic cross-section) which have non-co-linear axes - i.e. finite angular $\theta$ and axial offsets $d$ - as illustrated in figure 2 . In this appendix the derivation of equation (16) for the more simpler symmetric beam (circular cross-section and isotropic phase-front curvature) case will be shown in terms of the more accessible beam-tracing parameters. The coupling for some special cases will also be calculated.

A symmetric beam with a circular cross section $w_{\Psi, \text { maj }}=w_{\Psi, \text { min }}$ and isotropic phase-front curvature $R_{\Psi, \text { maj }}=R_{\Psi, \text { min }}$ is considered to hit the antenna plane with a finite angle $\theta \ll 1$ and an offset $d$. The Beam tracing matrix for the wave $\chi$ of the receiver antenna at the antenna opening can be written as:

$$
\sigma=2 / w_{\chi, 0}^{2}\left(\begin{array}{ccc}
0 & 0 & 0 \\
0 & 1 & 0 \\
0 & 0 & 1
\end{array}\right)
$$

In the frame of the receiver antenna the quantities of the incident wave $\Psi$ can be written e.g. as (compare also relations (11)):

$$
\begin{gathered}
\vec{q}=\left(\begin{array}{l}
0 \\
0 \\
d
\end{array}\right), \quad \vec{K}=\left(\begin{array}{c}
K \cos \theta \\
0 \\
K \sin \theta
\end{array}\right) \approx\left(\begin{array}{c}
K \\
0 \\
K \theta
\end{array}\right), \\
\Sigma=2 / w_{\Psi}^{2}+i K / R_{\Psi}\left(\begin{array}{ccc}
\sin ^{2} \theta & 0 & \sin \theta \cos \theta \\
0 & 1 & 0 \\
\sin \theta \cos \theta & 0 & \cos ^{2} \theta
\end{array}\right) \\
\approx \underbrace{2 / w_{\Psi}^{2}}_{\sim \phi}+i \underbrace{K / R_{\Psi}}_{\sim s}\left(\begin{array}{lll}
0 & 0 & 0 \\
0 & 1 & 0 \\
0 & 0 & 1
\end{array}\right)
\end{gathered}
$$

Building the determinant of the $2 \times 2$ yz-submatrix of $\Sigma+\sigma$ yields as the co-linear coupling:

$$
T_{0}=\frac{4}{\left(w_{\Psi} / w_{\chi, 0}+w_{\chi, 0} / w_{\Psi}\right)^{2}+K^{2} w_{\Psi}^{2} w_{\chi, 0}^{2} / 4 R_{\Psi}^{2}}
$$

The first exponential term follows as:

$$
\exp \left[-\phi_{\alpha \beta} q_{\alpha} q_{\beta}\right]=\exp \left[-2 d^{2} / w_{\Psi}^{2}\right] .
$$

To calculate the second exponential term in equation 15 the vector $\vec{b}$ has to be determined (only yz components):

$$
\vec{b}=\Sigma \cdot \vec{q}-i \vec{K} \approx\left[2 d \theta / w_{\Psi}^{2}+i K(d / R-\theta)\right]\left(\begin{array}{l}
0 \\
1
\end{array}\right)
$$

then, the second exponential term can be derived by building the inverse of the 2 x 2 (yz-components) submatrix of $\Sigma+\sigma$ (this is trivial since the matrix is proportional to $\mathbf{1}_{2}$ ), multiplying this from left and right with the vector $\vec{b}$ and taking the real part of 
this expression. Combining all these expressions finally yields equation (16), the coupling efficiency for a symmetric beam with finite angle of incidence and offset.

Consider now the case where the beam hits the antenna directly without an axial offset $(d=0)$ but at an angle $(\theta \neq 0)$, then equation (16) simplifies to:

$$
T_{a} \approx T_{0} \exp \left\{-T_{0} K^{2} \theta^{2}\left(w_{\chi, 0}^{2}+w_{\Psi}^{2}\right) / 8\right\}
$$

in agreement with equation 17 in [28].

For the case where the beam hits the antenna plane perpendicular $(\theta=0)$ but with an axial offset, equation 16 simplifies to:

$$
T_{a} \approx T_{0} \exp \left\{-T_{0} d^{2}\left[w_{\Psi}^{-2}+w_{\chi, 0}^{-2}+K^{2} w_{\Psi}^{2} / 4 R_{\Psi}^{2}\right] / 2\right\}
$$

in agreement with equation 20 in [28].

¿From equations (A.6) and (A.7) it can be seen that a finite angle of incidence or an axial offset in incidence lead to an exponential drop in the power coupling. If both offsets occur simultaneously then, as described by equation (16), the effects combine leading to an even stronger drop in coupling.

As already stated, the coupling efficiency for the general case of an asymmetric beam can be calculated with equation (15) without difficulty.

\section{References}

[1] C. Laviron, et al., Reflectometry techniques for density profile measurements on fusion plasmas, Plasma Phys. Control. Fusion 38 (1996) 905.

[2] G.D. Conway, Microwave reflectometry for fusion plasma diagnosis, Nucl. Fusion 46 (2006) S665.

[3] A. Donné, et al., Progress in the ITER Physics Basis, Chapter 7:Diagnostics, Nucl. Fusion 47 (2007) S337.

[4] G.Vayakis, et al., Status and prospects for mmwave reflectometry in ITER, Nucl. Fusion 46 (2006) S836.
[5] G.J. Kramer, et al., 2D reflectometer modelling for optimizing the ITER low-field side X-mode reflectometer system, Nucl. Fusion 46 (2006) S846.

[6] W.A. Peebles, et al., Assessment of ITER LFS Reflectometer System, presentation at BPO Diagnostics Workshop General Atomics, (2007).

[7] G. Wang, et al., Refractive and relativistic effects on ITER low field side reflectometer design, Rev. Sci. Intrum. 81 (2010) 10D908.

[8] G.R. Hanson, et al., Analysis of the ITER LFS reflectometer transmission line system, Rev. Sci. Intrum. 81 (2010) 10D920.

[9] J.J. Degnan, Waveguide Laser Mode Patterns in the Near and Far Field, App. Optics 12 (1973) 1026 .

[10] P.F. Goldsmith, Quasi-Optical Techniques, Proc. IEEE 80 (1992) 1729.

[11] G. Pereverzev, Paraxial WKB solution of a scalar wave equation, in: B. Kadomtsev (Ed.), Rev. Plasma Physics 19, Consultants Bureau, (1996).

[12] G. Pereverzev, Beam tracing in inhomogeneous anisotropic plasmas, Phys. Plasmas 5 (1998) 3529 .

[13] Y. Kravtsov, Y. Orlov, Geometrical Optics of inhomogeneous Media, vol. 6 of Springer Series on Wave Phenomena, Springer, Berlin, (1990).

[14] I.B. Bernstein, Geometric optics in space- and time-varying plasmas, Phys. Fluids 18 (1975) 320 .

[15] R.A. Cairns, et al. Calculation of a wave field from ray tracing, Nucl. Fusion 50 (2010) 095001.

[16] E. Poli, et al., Paraxial Gaussian wave beam propagation in an anisotropic inhomogeneous plasma, Phys. Plasmas 6 (1999) 5 .

[17] E. Poli, et al., EC beam tracing in fusion plasmas, Fus. Eng. Des. 53 (2001) 9. 
[18] A. Stegmeir, Anwendung der Beam Tracing Methode zur Diagnostik von Fusionsplasmen, diploma thesis at Technische Universität München, IPP report: 522652.0, (2010).

[19] A.R. Polevoi, et al., Requirements for pellet injection in ITER scenarios with enhanced particle confinement, Nucl. Fusion 45 (2005) 1451.

[20] E. Poli, et al., TORBEAM, a beam tracing code for electron-cyclotron waves in tokamak plasmas, Computer Physics Comm. 136 (2001) 90.

[21] E. Poli, et al., Boundary conditions for a Gaussian wave beam, Phys. Plasmas 8 (2001) 4325.

[22] E. Mazzucato, Relativistic Effects on microwave Reflectometry, Phys. Fluids B 4 (1992) 3460.

[23] H. Bindslev, Relativistic expressions for plasma cutoffs , Plasma Phys. Control. Fusion 35 (1993) 1093.

[24] O. Maj, et al., Validation of the paraxial beamtracing method in critical cases, Phys. Plasmas 16 (2009) 16.

[25] O. Maj, et al., Effects of aberration on paraxial wave beams: beam tracing versus quasioptical solutions, Plasma Phys. Control. Fusion 52 (2010) 085006.

[26] G.D. Conway, et al., Doppler reflectometry on ASDEX Upgrade: Foundations and latest results, Proc. 8th Intl. Reflectometry Workshop - IRW8 (St.Petersburg), http://plasma.ioffe.ru/irw8/proceedings (2007).

[27] H. Kogelnik, Coupling and conversion coefficients for optical modes, vol. 14 of Microwave research institute symposia, Polytechnic Press, New York, (1964).

[28] W.B. Joyce, B. DeLoach, Alignment of Gaussian beams, Applied Optics 23 (1984) 4188.

[29] S. Silver, H. M. James (Eds.), Microwave Antenna Theory and Design, IEE Electromagnetic Waves Series 19, Peter Peregrinus Ltd, London, (1984).
[30] M. Shimada, et al., Progress in the ITER Physics Basis, Chapter 1: Overview and Summary, Nucl. Fusion 47 (2007) S1.

[31] Y. Gribov, et al., ITER-FEAT Magnetic Configuration and Plasma Position/Shape Control in the Nominal PF Scenario , Proc. 18th Int. Conf. on Fusion Energy (2000), CD-ROM file ITERP/02.

[32] E. Strumberger, et al., Application of ThreeDimensional Codes to Tokamaks, 29th EPS Conf. on Plasma Phys. and Contr. Fusion, Montreux 26B (2002), P-2.090.

[33] E. Strumberger, et al., Numerical investigations of axisymmetric equilibria with current holes, Nucl. Fusion 44 (2004) 464.

[34] C. Angioni, et al, Particle pinch and collisionality in gyrokinetic simulations of tokamak plasma turbulence, Phys. Plasma 16 (2009) 060702.

[35] G. Ramponi, et al, Capabilities of the ITER Upper Launcher for ELMy-H mode plasmas at low magnetic fields, J. Phys. Conf. Series 25 (2005) 243.

[36] D. Shelukin, F09. ITER HFS reflectometer (main plasma). Front-end devices and transmission line losses, ITER Report 3ZGSE9, (2010).

[37] V.S. Udintsev, et al, System design description document (DDD) - Diagnostic Low Field Side Reflectometry DDD-PBS 55.F2, ITER Report 47QCBN, (2011).

[38] G. Vayakis, L. Zabeo, Z-range of ITER plasmas for LFS reflectometry, ITER Report 3TFDLN, (2010).

[39] P. Gourdain, W. Peebles, Assessment of microwave power flow for reflectometry measurements in tokamak plasmas, Plasm Phys. Control. Fusion 50 (2008) 025004.

[40] P. Gourdain, W. Peebles, Application of reflectometry power flow for magnetic field pitch angle measurements in tokamak plasmas, Rev. Sci. Instrum. 79 (2008) 10F102. 
[41] G.D. Conway, et al, Antenna configuration options for the ITER low-field-side LFS reflectometer, ITER Report 35AHBB, (2011). 